\title{
APURDUTM
}

Euskal ikerketen aldizkaria | Revue d'études basques |

Revista de estudios vascos | Basque studies review

$5 \mid 2000$

Numéro V

\section{Le chant et l'identification culturelle des Basques (1800-1950)}

\section{Natalie Morel-Borotra}

\section{OpenEdition}

\section{Journals}

Édition électronique

URL : http://journals.openedition.org/lapurdum/1352

DOI : 10.4000/lapurdum.1352

ISSN : 1965-0655

\section{Éditeur}

IKER

Édition imprimée

Date de publication : 1 octobre 2000

Pagination : 351-381

ISBN : 2-84127-161-7

ISSN : $1273-3830$

Référence électronique

Natalie Morel-Borotra, «Le chant et l'identification culturelle des Basques (1800-1950) », Lapurdum [En ligne], 5 | 2000, mis en ligne le 01 juin 2009, consulté le 01 juillet 2020. URL : http:// journals.openedition.org/lapurdum/1352; DOI : https://doi.org/10.4000/lapurdum.1352 


\title{
Le chant et l'identification culturelle des Basques (1800-1950)
}

\author{
Natalie Morel Borotra \\ Université Michel de Montaigne - Bordeaux III
}

Le chant est devenu aujourd'hui l'un des domaines les plus spontanément associés au Pays Basque, et il fait partie des idées courantes que le « chant basque » a toujours existé, ou du moins qu'il est l'une des caractéristiques majeures du Pays Basque depuis fort longtemps. Pourtant, un survol historique nous montre qu'il n'en est pas ainsi. Le musicographe F. J. Fétis, par exemple, définissant les termes d'air et de chanson, relève les " airs nationaux » de différents pays ou régions d'Europe et les " airs d'un caractère particulier " des différentes provinces de France sans mentionner les chants basques (Fétis, F. J., $1836: 290,311$ ). Ce terme lui-même, si banal aujourd'hui, ne se rencontre semble-t-il qu'à partir de 1834, et avec un sens particulier (un « chant basque » est un texte de chant historique), plus restreint que l'acception courante actuelle, qui englobe des pratiques et un répertoire variés. Ce n'est que vers le milieu du XIX siècle que l'on commence réellement à s'intéresser aux « chants basques » en tant que manifestations musicales et non plus seulement littéraires (et ce n'est que peu à peu que l'on en vient à le considérer comme une spécificité basque). Cet intérêt se manifeste par le biais de la musique savante, de l'attention portée au folklore musical, du développement d'une pratique musicale (l'orphéon, puis la chorale) et de la volonté de définir ou de créer une nationalité (artistique et/ou politique).

On voudrait donc essayer de montrer que les désignations ont évolué, les contenus ont évolué, au fur et à mesure que se constituait ce que nous appelons aujourd.'hui le "chant basque »-d'autant que ce terme recouvre deux réalités : le répertoire et la façon de chanter. C'est le discours sur le « chant basque » qui retiendra pour cela notre attention, et nous permettra de retracer cette évolution.

\section{Danse basque et chanson française}

Dans les premiers récits de voyage en Pays Basque, il est peu fait mention du chant : manque d'intérêt pour ce qui n'est pas considéré comme un art, barrière de la langue ? Toujours est-il que les mentions des pratiques vocales sont très rares et succinctes ${ }^{1}$. La danse, en revanche, est constamment décrite (elle partage par- 
fois avec le jeu de paume l'évocation des divertissements du pays), en particulier le saut basque qui est souvent présenté comme la danse nationale. La réputation des danseurs basques à Paris n'est plus à faire (ils ont même donné au ballet un "pas de Basque ») et l'on connaît la définition que Voltaire donne des Basques dans La Princesse de Babylone (1966 [1768] : 506) : «Ces peuples qui derneurent, ou plutôt qui sautent au pied des Pyrénées. "

Est-ce parce que la pratique du chant, ou plutôt de la « chanson » comme on dit alors, est jusqu'au XVIII ${ }^{\circ}$ siècle très répandue, en particulier en France ? J.-J. Rousseau, parmi tant d'autres, le constatait : " tous les étrangers conviennent de notre supériorité dans cet art (...), on peut assurer que l'humeur chansonnière est une des caractéristiques de la nation (...). Battant ou battu, dans l'abondance ou dans la disette, heureux ou malheureux, triste ou gai, [le Français] chante toujours, et l'on dirait que la chanson est l'expression naturelle de tous ses sentiments $»$.

Cette remarque est rapportée dans Dictionnaire de la conversation, (Héreau, E., 1833 : article «chanson »), qui enchaîne : « les choses se sont modifiées depuis, et l'on reconnaîtrait difficillement dans le Français d'aujourd'hui le portrait que Rousseau a tracé du Français d'autrefois $»^{2}$. Il aurait alors fallu quelques décennies pour que « l'humeur chansonnière » du Basque, restée égale à elle-même, apparaisse comme un fait digne d'être remarqué. Elle frappe en tout cas Frederick Link (1801 : 53), professeur à l'université de Rostock, par contraste avec la France qui ne chante plus : « dans les autres provinces de France, on entend rarement le petit peuple chanter, en tout cas beaucoup plus rarement qu'avant la Révolution ; mais ici des chansons retentissent de chaque vallée ». Les Français - et les Basques eux-mêmes - seront plus longs à le remarquer.

\section{De Pierre-Jean Garat aux Cantabres}

En 1783, Pierre-Jean Garat chante en euskara à la cour de Versailles, devant Marie-Antoinette dont il devient le professeur de chant (il faudrait voir en quels termes cet épisode est rapporté par les contemporains, et les raisons invoquées: le jeune autodidacte qui arrive de sa province chante d'abord des airs de son pays?). Il semble qu'Izar ederra et Aitarik ez dut aient été par la suite particulièrement appréciés par la reine. Cet intérêt de Garat nous est aujourd'hui connu par des annotations portées au XIX ${ }^{e}$ siècle ; l'écrivain Augustin Chaho note ainsi dans Biarritz entre les Pyrénées et l'Océan (1855:248) : « il excellait à chanter les airs naïfs des montagnes labourdines ». Presque un siècle après la prestation d.e Garat à Versailles, le ténor Pascal Lamazou publie un recueil, avec accompagnement de piano, où il donne deux mélodies comme étant composées par lui (Irulia et Charmangarria). Un autre collecteur, J. D. J. Sallaberry (1870 : 403), reprenant les assertions de Chaho (qui parle au même endroit d'un « air basque auquel on a accordé une petite place dans le recueil des vaudevilles français $*$ ), indique que Mendian zoinen eder a été popularisé par Garat et inclus dans la quatrième édition de La clé du caveau, recueil de chansons réunies par P. Capelle à partir de 1811. Son biographe Miel, enfin, écrit (1841) : «C'est à son retour de Madrid qu'il donna à Catel le charmant air basque qui sert de motif principal à l'ouverture de l'Auberge de Bagnères. " 
Les pièces dont le titre vient d'être cité correspondent bien au type de la romance de la fin du XVIII siècle : une investigation plus poussée, menée à partir de sources contemporaines, permettra de voir comment ces romances en langue basque, qui sont passées aujourd'hui dans le fond populaire, étaient nommées et. perçues à l'époque, et quel rôle éventuel elles ont pu jouer dans la constitution du «chant basque ».

Il ne semble pas en tout cas que, de son vivant, l'on fasse un lien entre la voix exceptionnelle de P. J. Garat et son origine basque (et bordelaise !), que celui-ci revendique : bien que quelques voyageurs tels que Madame d'Aulnoy (1874 [1679] : 6) aient noté les « très belles voix » des habitants du Sud-Ouest de la France ( «j'ai remarqué dans toute la Guyenne et vers Bayonne que l'on y a de la voix naturellement et il n'y manque que de bons maîtres »), les Basques n'ont sans doute pas acquis auprès du public la réputation de chanteurs qu'ils auront par la suite. En d'autres termes, le Basque chanteur, ou le chanteur basque, "n'existe pas " encore, mais quand on s'intéressera plus tard au chant basque, on ressortira la figure de Garat, en tant que première grande voix basque célèbre.

Dans la première moitié du XIX ${ }^{\circ}$ siècle, on commence cependant à trouver ici ou là mention des aptitudes vocales des Basques, mais sans que ce point soit développé. Deux ans après la mort de Garat, l'abbé d'Iharce de Bidassouet, dans son Histoire des Cantabres ou des premiers colons de toute l'Europe, avec celle des Basques, leurs descendants directs, qui existent encore, et leur langue asiatiquebasque (1825: 12), donne l'étymologie suivante : «Cantabre, en basque khanta ber, chantre, chanteur sans pareil », avec en note : "les Romains les appeloient Cantabri, à raison de l'excellence de leurs voix ; aussi étoient-ils les ornements de leurs théâtres, comme le célèbre Garat, Basque, l'a été de ceux de Paris ». Cette référence romaine est même plus ancienne, puisqu'on la trouve au dernier distique de la dédicace faite par Etcheberri de Ciboure à Bertrand d'Etchauz de son Eliçara erabilceco liburua (1636 : " ecen daquiçun beçala Escualdunac, çaharrec,/ Cantuen maitatçaille deithu zituzten Erromarec » : «car comme vous le savez, les anciens Romains nommèrent les Basques amateurs des chants "), ou encore au XVII siècle sous la plume de Jacques de Bela ( 1 je tiens que le mot Cantaber fut donné aux Basques pour ce qu'ils sont joviaux et chanteurs de leur nature $\aleph^{3}$ ).

Cette étymologie sera reprise dans plusieurs dictionnaires, ce qui contribuera sans doute à la diffusion de l'idée. On la trouve par exemple dans le Dictionnaire de la conversation de 1833 (Garay de Monglave, E. : article « Basques», avec allusion en forme de "preuve » aux chanteurs d'opéras tels que Garat, comme chez d'Iharce), mais pas dans l'édition de 1873, ou dans le Dictionnaire universel Larousse de 1867 ([s. n]. : article "Basques »). Le voyageur anglais George Borrow (1989 [1845] : 264-265) la cite encore et ajoute : « Les Basques sont plus portés à la musique qu'à la poésie, et bien que la composition des vers leur soit facile, ils y réussissent assez mal. Par contre, leurs voix sont particulièrement agréables et leurs compositions musicales surpassent en beauté celles des autres peuples. » 


\section{Une " découverte » allemande}

C'est un Allemand qui, le premier, s'intéresse au « chant basque », et non pas seulement à la « voix basque ». Les Allemands sont peut-être plus sensibilisés à la musique que leurs contemporains venant d'autres horizons culturels. De plus, leur intérêt pour le populaire, où ils voient s'incarner «l'esprit national », « l'âme du peuple ", a été particulièrement aiguisé dans le domaine qui nous intéresse par la publication par J. G. Herder, en 1778-1779, de son recueil de Volkslieder ("Chansons populaires »), réédité sous le titre Stimmen der Völker in Liedern ("Voix des peuples dans leurs chansons "). À la génération suivante, Clemens Brentano et Achim von Arnim poursuivent sa quête de « chansons populaires », et font paraître en 1806 Das Knaben Wunderhorn ("Le cor enchanté de l'enfant »). Seuls les textes sont donnés (la première compilation avec musique date de 1840 : il s'agit des Deutsche Volkslieder de A. Kretzschmer et A. von Zuccalmaglio) ; on ne discutera pas ici de leur authenticité ou des transformations que les « collecteurs " ont pu leur faire subir, seul nous importe le retentissement de ces publications - qui n'est pas limité aux musiciens et aux poètes.

Wilhelm von Humboldt, ambassadeur du roi de Prusse, visite le Pays Basque en 1799 et 1800 , et même s'il est animé d'objectifs historiques et linguistiques, il ne néglige pas pour autant les aspects « culturels» du pays ${ }^{4}$, notamment « ses proverbes, ses danses nationales, sa musique et sa poésie » (Humboldt, W. von, 1975 [1801] : 20). Ceux-ci sont les témoins du «mode de pensée et de vie» des Basques dont il veut également rendre compte et quil l'aideront, pense-t-il, dans ses « investigations scientifiques sur l'origine de la nation basque et de sa langue ». W. von Humboldt distingue également les Souletins, «Italiens parni les Basques », pour leur inclination particulière pour la poésie et la musique (1975: 185-186). S'il a entrepris ce voyage, $c$ 'est afin de « chercher les traces des plus vieilles constitutions du peuple et des p us vieilles histoires à trouver toujours dans les anciens dictons et chants nationaux », précise-t-il ailleurs (Bidart, P., $1997: 318$ ).

Jorge de Riezu a publié, sous le titre de Papeles de Humboldt, des documents trouvés à la Preussische Staatsbibliothek de Berlin, comprenant quelques pages en espagnol datées de 1802 environ et intitulées De la Música en el País Vascongado, des partitions de musique de danse ainsi que trois petites pièces arrangées à quatre voix et des textes poétiques. De la Música... est attribué à Juan. Antonio Moguel, prêtre biscayen qui fut l'un des « informateurs » de W. von Humboldt. Malheureusement, la détérioration du manuscrit le rend partiellement illisible. Son auteur y présente le Zorzico ${ }^{5}$ [sic], qu'il soit vocal ou instrumental, et le Contrapas comme les « cantilènes propres" (cantilenas privativas) du Pays Basque. Il semble vouloir insister sur l'originalité du zortziko, et termine par une remarque sur l'" heureuse disposition [des Basques] pour la musique en général, et [leur] extrême sensibilité pour la leur en particulier " (Riezu, J. de, 1994 : 1529-1536). Les textes poétiques sont en basque, écrits de la main d'Humboldt et groupés sous deux intitulés allemands : Lieder deren sich der 78 jährige Harambillet in Itzazu aus seiner Juengend erinnerte (chansons de sa jeunesse qu'Harambillet d'Itsassou se rappelait à 78 ans) et Durch Ithurbide in Bayonne erhaltene Gedichte (poésies 
recueillies à Bayonne grâce à Ithurbide). Il semble donc qu'Humboldt se soit adonné lui-même au collectage (limité au texte, comme Herder). Certaines des "poésies » de Bayonne portent un titre français : Chanson basquaise, Chanson libre, Chanson bachique (2), Lamentation d'un vieillard, La rencontre du créancier et du débiteur (op. cit. : 1553 et ss.).

Ces documents n'ont pas été exploités jusqu'en 1934, date à laquelle le Père Donostia en a appris l'existence. En revanche, W. von Humboldt publie lui-même, en 1817, en faisant quelques réserves, le texte du Chant de Lelo. C"est la première publication d'un " chant basque » (et, avec les deux textes accompagnés d'une traduction française donnés par E. Boucher de Crèvecœur en 1823 dans ses Souvenirs du Pays Basque et des Pyrénées, qui seront repris dans des publications allemandes en 1825 et 1831, la seule jusqu'aux années 1830 , si l'on en croit la bibliographie établie par Julien Vinson en $1884^{6}$ ). Elle va certainement contribuer à orienter l'intérêt vers un certain type de chant : ce Chant de Lelo est censé raconter la résistance des Cantabres, considérés comme les ancêtres des Basques, par les armées romaines commandées par Auguste, et se révélera plus tard comme n'étant pas du tout contemporain des événements qu'il met en scène.

\section{Les " chants nationaux basques "}

Les Basques, en cette première moitié du XIX' siècle, commencent à être l'objet d'études dans tous les domaines (sang, mœurs, langue, usages, pour reprendre par exemple les rubriques énumérées à propos de la spécificité qu'on leur attribue dans le Dictionnaire de la conversation en 1833, Garay de Monglave, E. : article « Basques »). On s'intéresse à la littérature : or la littérature écrite apparaît presque inexistante; on se tourne alors vers la littérature orale, et on la trouve dans les chants, et tout d'abord les " chants nationaux », qui représentent donc la première forme reconnue que prend le " chant basque ». Ces deux termes sont employés dès 1834 dans le Dictionnaire de la conversation (Olivier, G., 1834 : article « chants populaires ») et l'idée se rencontre dès lors couramment : « la vraie littérature basque se trouve dans les chants nationaux », écrit A. Mazure (1839: 519), professeur de philosophie au lycée de Pau et auteur d'une Histoire du Béarn et du Pays Basque. Cette quête de chants nationaux, c'est-à-dire historiques (l'histoire restant une discipline reine dans la hiérarchie des savoirs), est surtout perceptible en France, qui se sent inférieure, dans ce domaine, à l'Angleterre, l'Allemagne et l'Espagne ${ }^{7}$, et on trouve encore la catégorie " chants historiques » dans la nomenclature établie pour l'enquête Fortoul en $1853^{8}$.

Dans les provinces basques d'Espagne, un intérêt pour la langue se manifeste au XVIII et au début du X.IX siècle, avec un retentissement ibérique et parfois international (il est le fait des « apologistes » tels que Larramendi, Astarloa, Moguel), puis on observe un retrait de la scène intellectuelle dû aux guerres carlistes et, à partir de 1850, un goût davantage porté vers le roman historique et la légende que vers le chant : dans la première moitié du XIX siècle, le " chant basque » est donc essentiellement une « invention » de linguistes allemands, de voyageurs anglais, de « savants » français et d'hommes de lettres originaires du Pays Basque (d'expression française). 
De plus, il y a l'idée que « la chanson » est à la base de tous les genres littéraires (la chanson, « poésie naturelle du peuple», " au lieu d'être un. genre, est le: berceau de tous les genres » explique $\mathrm{H}$. Fortoul dans l'Encyclopédie nouvelle, 1841 : article " chanson ») et que la première forme sous laquelle elle se manifeste généralement est le genre épique (id.). Les Basques ont-ils eu des productions relevant de ce domaine ? Ils « avaient indubitablement une poésie », estime l'historien. Claude Fauriel (1836:353), « et quelques rudiments des arts immédiatement liés avec elle, tels que la musique et la danse. Le nom d'eressiac [sic], dont ils se servent encore pour caractériser les chants populaires qui roulent sur quelque histoire vieille ou antique, a l'air d'être fort ancien dans la langue, bien que les pièces de: poésies auxquelles il peut s'appliquer soient toutes assez modernes ».

Quoi qu'il en soit, on va donc rechercher les chants historiques, qui seront appréciés pour leur ancienneté (le goût est aux « antiquités 》 dans tous les domaines depuis le début du siècle) et leur intérêt d'information et de témoignage historiques : jusqu'aux années 1860 au moins, c'est la vogue des chants historiques, dont les plus célèbres s'avèrent apocryphes (c'est le cas, par exemple, du Chant de Lelo, du Chant d'Altabiscar « fabriqué » en 1834 par le littérateur Eugène Garay de Monglave à partir d'une énumération de nombres se récitant de façon traditionnelle, du Chant d'Annibal inventé par A. Chaho en 1845, etc.). Dans l'édition de 1878 du Dictionnaire de la conversation (article " chants populaires»), G. Olivier s'émerveille : « on possède une centaine de chants de guerre euscariens ». Les chants nationaux sont donc ces « monuments de la vieille langue des Escualdunacs » (A. du Mège, 1858, cité par J. Vinson 1884: 44), qui vont permettre de mieux saisir un peuple et une histoire encore très mal connus. Les études basques n'en sont qu'à leurs balbutiements, dans tous les domaines, et tant dans les encyclopédies et dictionnaires français que chez les auteurs locaux, on trouve des remarques soulignant ces lacunes, le regrettant parfois : « l'histoire politique, physique, intellectuelle et pittoresque des Basques n'a pas été racontée. C'est une mine d'or qui demande à être exploitée » (Morel, F., 1836:501).

On comprend donc que les chants n'intéressent pas vraiment en eux-mêmes, mais par les renseignements qu'ils apportent comme le souligneront encore les Instructions d'Ampère en 1853, il s'agit "d'éclairer les parties obscures de nos annales » (Cheyronnaud, J., 1997:83). Les publications éparses de chants isolés, dans des ouvrages plus généraux, et dans des revues (Album pyrénéen, Ariel en particulier), n'en donnent par conséquent que le texte. La traduction revêt une importance primordiale, car le débat est international - et le basque une langue particulièrement hermétique.

\section{Le " chant populaire",}

\section{" tableau des mœurs et du caractère de la nation basque "}

La notion de « chant basque » va ensuite s'élargir au « chant populaire », tel que le définit le Dictionnaire de la conversation (Olivier G., 1833 : article « chants populaires ») : «le chant populaire, c'est celui qui, sans relation directe avec aucun 
paroxysme donné du patriotisme, se montre cependant le fils le plus dévoué de la patrie, qui en revêt les mœurs, en garde les coutumes, et se fait l'arche dépositaire de ses plus précieux souvenirs ; c'est celui qui n'oubliera jamais les conquêtes ni les croyances de nos aïeux ; c'est la ronde de noce, la chanson de berceau, de table ou de métier (...) c'est enfin toute mélodie qui porte empreints la nationalité d'un peuple, ses mœurs, ses jeux, ses usages, ses traditions et ses croyances ».

National encore bien sûr, mais plus seulement historique et guerrier, il rend compte d'une culture (dans l'acception large du terme). A. Mazure l'avait dit, à propos des Basques (1839:520): « interroger [sic] les vieux souvenirs de ce peuple antique [en recueillant les chants] ; ils ne seront point ingrats ; peut-être révéleront-ils des traditions que l'on pouvait croire évanouies depuis des siècles ". Il ajoutait : « là est l'homme, là est le peuple ». Quelques années plus tard, A. Chaho, publiant une « improvisation populaire » (voir plus loin) dont il donnait le titre en français : Amour et devoir, la voyait occuper « une des premières places dans le tableau des mœurs et du caractère de la nation basque » $\left(1845, \mathrm{n}^{\circ} 30\right.$ : «Poésie cantabre $\gg)$.

Le terme de chant populaire n'est cependant pas le plus répandu dans les années 1830 , on lui préfère - pendant une trentaine d'années - celui de « poésie populaire » mettant en reliefle primat donné au texte (cf. les Instructions pour un Recueil général de poésies populaires de la France qui encadrent l'enquête Fortoul par exemple). L'intérêt pour le texte seul perdure dans les fêtes basques instituées par Antoine d'Abbadie d'Arrast, comportant un «concours de poésie » à partir de 1853 : la «chanson » primée est en fait une poésie primée, chantée sur un air connu qui n'est qu'un support; le chant n'est alors qu'un moyen de rendre publique la poésie couronnée. Une lettre adressée par le chanoine Harriet à d'Abbadie le 3 juin 1853 expose clairement la situation : « il faut populariser l'œuvre dès le principe ; par suite adopter une forme que généralement on comprenne ; or le: Basque n'entend du sentiment et de la poésie qu'autant qu'il en chante ; c'est du reste le privilège des peuples qui en sont encore à la naïveté de la poésie primitive ; ils aiment, ils souffrent, ils jouissent en chantant. Ne faudrait-il pas que les pièces fussent des chansons ? (...) Ne serait-il pas utile d'exiger un air connu de tous, un de ces airs populaires que nos montagnards savent depuis longtemps et entendent toujours avec plaisir ? $\aleph^{10}$. En réalité, l'air ne sera pas imposé, mais laissé au choix du candidat - à moins que celui-ci ne le laisse à la liberté du jury... On peut compléter l'analyse des relations entre poésie et « chant basque » en rappelant que la poésie écrite peut être à l'origine d'une pièce passée dans le domaine populaire, comme c'est le cas par exemple de la très célèbre chanson Nere etchea edo laboraria, pour laquelle Jean-Baptiste Elissamburu obtint le prix de poésie à Urrugne en 1862 : selon Madame de la Villehélio" ", la poésie est chantée sur un zortziko préexistant, dont elle donne une version pour piano (1869:23), et elle est déjà très répandue sept ans après son écriture.

La terminologie pour ce qui ne concerne pas les chants nationaux ou populaires au sens de chants historiques est variée : «ballade», " chansonnette », "romance» (Garay de Monglave, E., 1833 : article « Basques »), « complainte », « légende », 
«élégie » (Chaho, A., 1845 : Ariel, passim), « sérénade », « nocturne » (Sallaberry, J., 1870 : passim), ces termes reprenant des catégories de la poésie et de la musique savantes (elles-mêmes pouvant être d'inspiration populaire, comme la ballade).

\section{Vers une nouvelle esthétique littéraire}

Chants historiques, chansons politiques, légendes poétiques, chants funèbres, romances, chants de Montevideo [c'est-à-dire concernant l'émigration en Amérique du Sud], chansons morales, satires, chansons diverses - pour reprendre par exemple les rubriques définies par Francisque-Michel en 1857 font donc l'objet d'études et de rapprochements à partir des années 1830 (est-ce l'article d'E. Garay de Monglave, donnant en 1834 dans le Journal de l'Institut historique le texte du Chant d'Altabiscar, qui « lance » véritablement le «chant basque » sur la scène intellectuelle?).

Des polémiques éclatent au sujet de leur authenticité et de leur ancienneté (surtout pour les chants historiques ${ }^{12}$ ), ou de leur valeur littéraire, qui est loin d'être une évidence : « on demandera peut-être si les Basques ne possèdent pas des poésies populaires, comme la plupart des autres nations, quelque petites et peu considérables qu'elles soient. Certainement ils ne sont point dépourvus de chansons, de ballades ni de couplets; mais ces pièces ne présentent aucun caractère qui mérite le nom de poésie », estime par exemple G. Borrow en $1843^{13}$. II rejoint C. Fauriel $(1836: 525)$ : « pour ce qui est des chants modernes [c'est-à-dire ceux qui ne sont pas des chants historiques ?] des Basques, je n'en connais pas qui méritent d'être cités, et j'ai entendu dire la même chose par des Basques lettrés ». A. Mazure, en. revanche, trouvait le « tour d'imagination mélancolique et narratif du peuple basque (...) remarquable » dans les " chansons basques » des vallées de Soule et: de Baïgorry (1839: 518).

Point de vue érudit, étude par des « savants ", appréciation dans la triple perspective historique, ethnographique et littéraire : tel est donc le cadre intellectuel à l'intérieur duquel s'élabore la notion de " chant basque » dans la première moitié du XIX ${ }^{\circ}$ siècle. En ce qui concerne ce dernier point, l'intérêt pour le "chant basque " (et pour le « chant populaire» en général) s'inscrit dans un renouvellement non. seulement du goût littéraire, mais aussi des catégories et des méthodes employées dans les études littéraires, en particulier en. France. La démarche intellectuelle et la carrière de Claude Fauriel les illustrent bien : une chaire de littérature moderne étrangère (spécialité alors inconnue en. France) est créée pour lui à la Sorbonne (1830), par laquelle il manifeste son intérêt pour les études comparées, et pour la recherche des " origines de la civilisation moderne » dans les cultures jusque là délaissées : arabe, provençale, celte, basque, etc.

L'Antiquité gréco-latine cesse d'être considérée comme la référence et la source uniques, le classicisme n'impose plus sa loi, ce qui suppose et suscite une évolution des canons esthétiques, et conduit à une valorisation des caractéristiques du « populaire » : « limpidité, spontanéité, fécondité, fraîcheur » (Parisot et anonyme, 1855 : article «Fauriel») sont par exemple les qualités reconnues aux Chants 
populaires de la Grèce moderne que C. Fauriel traduit et publie en 1824, et qui marquent une étape importante, par leur retentissement, dans l'évolution du goût en littérature.

Ballades anglaises, chants populaires écossais, romances espagnols, chants serbes sont également traduits en français et assez largement diffusés à la même époque. En France, une collecte organisée a du mal à se mettre en place, malgré les efforts de l'Académie celtique, puis de la Société Royale des Antiquaires de France, et du projet Salvandy en 1845 : il faut attendre l'enquête Fortoul, dont on verra plus loin les (maigres) résultats en ce qui concerne le Pays Basque. L'introduction de Francisque-Michel à son étude sur les « poésies populaires des Basques » permet cependant de mesurer l'évolution de l'attitude envers la littérature populaire. Il commence en citant Montaigne (citation souvent utilisée dans ce contexte, par exemple dans les Instructions d'Ampère) : " la poésie populere et purement naturelle a des naïfvetez et graces par où elle se compare à la principale beauté de la poésie parfaite selon l'art : comme il se voit ès villanelles de Gascouigne (...) » (Francisque-Michel $1857: 209)$; puis il rejoint A. Mazure et A. Chaho, en lui trouvant un intérêt " pour ainsi dire psychologique »: ses productions « révèlent les aventures privées d'un peuple, les allures de son caractère, les attitudes de son esprit : ce sont des mémoires, ou plutôt des confessions ». Il conclut alors : " après cela, il n'y a pas à douter que la poésie populaire ne devînt une source féconde et réparatrice pour la poésie d'art » (op. cit. : 211). Même si les « chansons basques » lui paraissent inférieures aux pièces serbes et bretonnes, il y trouve «des inspirations heureuses, des élans vraiment poétiques» (ibid. : 221).

\section{"Un vrai chant primitif »}

En ce qui concerne le Pays Basque, C. Fauriel (1836:353) parle en ces termes du « chant cantabre " publié par Humboldt (Chant de Lelo) : il lui apparaît « très curieux, ne fût-ce que par la rudesse sauvage de ton et de style qui le caractérise. C'est, dans toute la propriété du terme, un chant de montagnard, un vrai chant primitif, où l'art en est encore aux plus simples inspirations de la nature ".. Primitivisme, " naturalisme » rousseauiste, enfance de l'art correspondant à une enfance du peuple et de la civilisation, ces idées se rencontrent fréquemment pour caractériser le chant populaire, et particulièrement le chant basque (à cause du rôle de la montagne, sur lequel on reviendra plus loin, et de la situation et la spécificité des populations basques, qui forment un peuple singulier, retranché du reste du monde, etc ${ }^{14}$ ?), connotées positivement ou négativement selon les options intellectuelles et l'origine de celui qui les transcrit.

Ainsi peut-on lire dans l'Encyclopédie des gens du monde (de Walckenaer 1834 : article « Basques (peuple et langage) 》), une condamnation sans appel de ces « quelques petites chansons satiriques » dont « aucune n'a mérité d'être écrite " : " chez aucune des branches de la nation basque, soit en Espagne, soit en France, il n'existe de vestige d'une littérature qui soit propre à ce peuple très ignorant ", peuple qui « a toutes les qualités et tous les défauts attachés à un état social qui participe du sauvage et de l'homme civilisé ». A. Chaho en revanche (1844 : 
" Chants basques »), après avoir évoqué la chaîne non rompue des « traditions primitives ", estime : " nation neuve encore, malgré sa haute antiquité, les Basques sont restés près de la nature, et ils doivent à cet avantage une certaine hardiesse de conception et d'entreprises qui les distingue, et surtout le don de l'invention poétique $»$.

Dans l'Encyclopédie des gens du monde (Spach, L., 1835 : article « chants populaires »), Louis Spach, après avoir disserté sur le peuple qui « chante comme le vent souffle, comme le ruisseau murmure » et passé en revue la situation des différents pays européens dans ce domaine, s'intéresse au cas de la France ; le seul exemple qu'il cite concerne le chant basque : « la France est peut-être moins riche que d'autres pays en chants primitifs [...]. Cependant, si l'on s'appliquait sérieusement à recueillir dans toutes les provinces ces voix perdues du passé [...] la moisson serait plus riche qu'on ne pense. Les montagnes surtout recèlent de curieuses mélodies, accompagnement de paroles bizarres. Au fond des Pyrénées, le descendant des Basques a conservé de mélancoliques chansons dont il accompagne sa danse ou dont il charme sa solitude ». De telles remarques, concernant aussi la musique, sont rares. Aussi la parution du recueil d'Iztueta fait-elle figure d'exception.

\section{Un premier " monument national "}

Le recueil publié par Juan Ignacio de Iztueta (matelassier de son état, mais aussi auteur d'une histoire de sa province natale) à Saint-Sébastien dément à première vue le fait qu'on ne s'intéresse pas encore à l'aspect musical. Guipuzcoaco dantza gogoangarrien condaira edo historia beren sonu zar, eta itz neurtu edo versoaquin, c'est-à-dire « Notice ou histoire des danses les plus mémorables du Guipuzcoa, avec les airs anciens et les paroles mesurées ou vers » (1824), est suivi, deux ans plus tard, d'un autre ouvrage présentant la musique de ces danses: Euscaldun ancina ancinaco ta lendabicico etorquien dantza on iritci pozcarri gaizic gabecoen sonu gogoangarriac beren itz neurtu edo versoaquin, « Des anciens Basques et de leur première origine, de leurs danses aimées et sans tache, les airs mémorables avec leurs vers correspondants » contient une cinquantaine de numéros, dont trente danses avec paroles, notées grâce à la collaboration de l'organiste d'Ernani, Manuel de Larrarte, et d'un compositeur basque disciple de Rossini, Pedro Albéniz.

Mais la lecture de la préface de l'édition de 1826 situe encore le travail d'Iztueta dans une perspective avant tout intellectuelle. Iztueta, en effet, voit dans ce qu'il nomme coleccion de canciones bascongadas ou coleccion de cantos proprios del pais [sic] une contribution à une étude historique de type comparatiste, destinée à mettre au jour les échanges, les « anciennes communications entre des peuples très éloignés les uns des autres » Or les historiens s'intéressent essentiellement aux affrontements armés, « tout au plus [au récit] de certains attributs caractéristiques du naturel des peuples ", et lui (Iztueta) souhaite que le champ d'étude soit élargi aux modes de vie, aux danses et aux chants - d'où son ouvrage. 
Pour le cas où cet objectif de " comparer les traditions musicales des peuples " paraîtrait « trop élevé et philosophique ", Iztueta se console en pensant " avoir fait une œuvre chère au peuple basque en sauvant de l'oubli ces chansons dont sûrement une grande partie compte des siècles d'ancienneté ». Comment faut-il entendre le fait de « sauver de l'oubli » ce répertoire ? Etait-il en voie d'extinction? (cela ne ressort pas toujours des autres propos d'Iztueta). Ou faut-il comprendre que le fait d'être publié par l'imprimerie musicale (procédé nouveau en Guipuzcoa, et « peu répandu en Espagne ») val lui permettre de perdurer et de se faire connaître ? La fin de la préface est également un peu sibylline : Iztueta demande que son recueil ne soit pas regardé « seulement comme un objet de passetemps, mais comme un véritable monument national qui a et doit avoir plus d'importance que celle qu'il paraît peut-être avoir à première vue » : c'est-à-dire ? En tous cas, les chants publiés n'ont pas à ses yeux de valeur artistique : "prétendre [trouver] dans les chants vulgaires les combinaisons sublimes de l'art, serait une erreur grossière », écrit-il. Cependant, quand ces chants vulgaires présentent certaines qualités, cela montre que les peuples qui en sont l'auteur ont des dispositions " pour l'art enchanteur de l'harmonie " - et on comprend qu'Iztueta range les Basques parmi ces peuples-là. La mention qu'il fait ensuite de quelques compositeurs guipuzcoans laisse à penser que c'est une affirmation qui lui tient à cœur.

\section{Une musique étrange et déroutante}

G. Borrow (1989 [1845] : 265) connaît le recueil d'Iztueta, et voit dans certains de ses airs, " exemples assez curieux " de " compositions musicales fort anciennes », " des marches bruyantes et sauvages, probablement composées dans les temps où les Basques descendaient avec fureur de leurs montagnes pour repousser les Romains ou les Maures. Il semble qu'on entende les pas précipités de la cavalerie, le cliquetis des armes et le sourd roulement des multitudes précipitées du haut des monts cornme autant d'avalanches ». Rappelons que le recueil d'Iztueta contient des airs de danse encore largement connus aujourd'hui, et que les oreilles modernes n'y perçoivent pas tout ce que Borrow y entendait... Son appréciation est à rapprocher de la description de la danse contenue dans l'Encyclopédie catholique (M., J. de, 1842 : article « Basques (Provinces) (géographie) ») : « musique discordante et sauvage à faire frémir un homme à l'oreille délicate ».

F. Morel (1836 : 437), rédacteur de La Sentinelle des Pyrénées, note que lors de la fête des Basques à Biarritz, les femmes chantent " parfois en langue basque des airs plaintifs et monotones qui ne manquent pas pourtant d'harmonie ». Pour Francisque-Michel (1857: 435-437), qui émet (peut-être le premier en ce qui concerne spécifiquement le Pays Basque ${ }^{15}$ ?) l'idée qu'on ne peut séparer paroles et musique quand on juge un chant, G. Borrow fait du tort aux «chansons basques » (comme il les appelle) avec ses affirmations: selon lui, certaines pièces sont au contraire pleines d'intérêt. Mais le " jeune musicien d'avenir » (un certain George Amét ${ }^{6}$ ) à qui il s'est adressé pour une appréciation compétente est perplexe : «Dans certains de ces chants, le vague et la bizarrerie sont tels que j'ai cru avoir devant les yeux de véritables énigmes. » 
De tout cela on retiendra que, même si l'on reconnaît aux Basques des aptitudes musicales, les productions populaires dans ce domaine déroutent encore (jugées ou appréciées à l'aune de la musique savante, et donc soit inférieures par nature, soit « incompréhensibles » selon les critères « artistiques » de celle-ci). Pas plus que pour l'appréciation littéraires des poèmes, les chansons basques n'entraînent un jugement largement positif sur le plan musical. L'enthousiasme sans réserve manifesté par G. Olivier apparaît comme une exception : "d'où vient à ces tribus exilées entre ciel et terre une telle franchise de rythme et d'intonation. ?Tout ce que je connais d'airs basques est d'un ton grandiose et décidé » (Dictionnaire de la conversation, 1833 : article « chants populaires »).

\section{La médiation instrumentale}

Comme on vient de le voir, les remarques ou appréciations concernant la musique des pièces qui sont l'objet des travaux érudits sont donc rares et brèves. Plus rare encore le souci exprimé de recueillir la musique, et non plus seulement le texte des chants, comme le fait A. Mazure (1839:520): « il faut les [les chants nationaux] recueillir avec les mélodies expressives et variées qui les accompagnent ». Henry Wilkinson, chirurgien de l'armée britannique auteur d'un récit de voyage intitulé Sketches of Scenery in the basque provinces of Spain, with a selection of national music, arranged for piano-forte and guitar (Londres, 1838), est quant à lui unique à cette date en ce qui concerne le Pays Basque par sa curiosité intellectuelle, son appréciation de cette " musique nationale » et la publication de 32 pages de musique : sous l'intitulé « selection of Spanish music », plusieurs «zorcico, basque air », avec paroles basques ou anglaises et accompagnement instrumental (réalisé par M. Webster, de Glasgow), comme on en trouvera trente ans plus taid ${ }^{17}$. Dans sa préface, il donne ces précisions : « la musique contenue dans le présent volume comprend des spécimens de la plupart des mélodies nationales d'Espagne, telles qu'elles se manifestent dans les Hotas, Fandangos et Boleros ; mais l'auteur est certain qu' il innovera grandement avec quelques Zorcicos, ou airs d'une accentuation et d'un caractère particuliers, confinés exclusivement dans les provinces d'Alava et Guipuscoa. Il craint que ces dernières très belles mélodies perdent considérablement par leur adaptation anglaise. La langue à laquelle elles ont été jusqu'ici associées est le basque ou bascuense, un dialecte aussi différent du pur castillan que le gallois de l'anglais. Entendu dans une contrée sauvage, au milieu des œuvres sublimes de la Nature, et jaillissant sans art de groupes d'enfants, ces airs possédaient un charme indescriptible, et produisaient un effet qu'il serait illusoire d'essayer d'imiter dans un salon anglais. Toutefois l'Auteur affirme leur popularité parmi tous les bons musiciens. Comme mélodies elles ont de nombreuses particularités, et elles soutiendront la comparaison avec celles de n'importe quel pays [que ce soit] en beauté ou en originalité ».

Si c'est le texte qui amène les chercheurs, puis le public lettré, à s'intéresser à ces « chansons basques », c'est peut-être par la musique instrumentale que l'on va d'abord prêter attention à leur mélodie, donner à celle-ci une audience plus large et se mettre à l'apprécier. H. Wilkinson donne d'ailleurs un «zortzico» dans un 
arrangement pour flûte et piano ou guitare. La presse nous en apporte aussi des témoignages : «à la fin du concert, un air basque a été demandé ; quelque fatigué que fût Alard, il s'est joyeusement et résolument remis sur son violon; et après quelques riches variations, l'air basque est venu, et tous les visages Bayonnais se sont déridés, et les mains ont battu avec plus de retentissement encore » ([s. n], $\mathrm{La}$ Sentinelle des Pyrénées, 1841 : "Concert de M. Alard 》). Lee même scénario se répète à chacune des prestations dans sa ville natale de ce Bayonnais qui est devenu l'un des maîtres de l'école française de violon, et il joue également ce répertoire lors de concerts à Paris.

Cet air basque est-il Donostiako iru damatxo dont François Habeneck (qui fut son professeur au Conservatoire de Paris) a écrit des variations ${ }^{18}$ ? Ou plus vraisenblablement le Zorzico [sic], « air basque nouveau » qu'il donne lors de son second concert à Bayonne cette année-là ([s. n], Le Furet, 1841 : [Programme du concert]) ? À moins qu'il n'ait déjà à son programme cette " réunion d'airs nationaux, enchaînés avec art » dont il est fait mention en 1857 (Henry, A. : « Lettres à un ami »). La Sentinelle des Pyrénées explique en tout cas les raisons de son succès : « chaque pays a ses airs nationaux : donnez-lui la musique la plus savante ; étonnez-le, ravissez-le par toutes les ressources de l'art le plus parfait ; vous aurez son admiration, ses applaudissements, vous l'aurez ému, vous ne l'aurez point touché ; il lui faut l'air connu, l'air appris dès l'enfance, répété et jamais oublié » \op. cit.).

Ainsi donc le " chant basque » se fraie un chemin sur les scènes de concert, en tant que support (et prétexte ?) à la virtuosité instrumentale ${ }^{19}$, dans un genre très pratiqué et appréciè à cette époque (la variation sur un thème connu ou donné), auquel la provenance basque ajoute un peu de pittoresque ou de nostalgie sentimentale. On peut penser que la mise en lumière de cet aspect du chant basque a joué un rôle non négligeable dans la reconnaissance de ce chant comme production musicale et non plus seulement littéraire.

Remarquons que l'emploi du terme " air basque », que l'on retrouvera très régulièrement dans la presse bayonnaise lors des prestations de Delphin Alard, y est appliqué à ce que nous nommons aujourd'hui chant populaire basque pendant au. moins une trentaine d'années : en 1854, P. Lamazou chante "Aiñhara, air basque " (Moncla, J., 1854 : " Théâtre de Bayonne ») ; en 1860, les frères Lionnet, chansonniers parisiens dont la mère est luzienne, chantent "d'une manière charmante » un « air basque bien connu, qui ne faisait pas partie du programme » mais qui leur a été demandé (" c'est Chapela gorria », in Le Courrier de Bayonne, 1860 : [sans titre]), etc.

Ce terme est très couramment employé au XVIII ${ }^{e}$ siècle. Il est lié à la définition même de la "chanson", telle qu'on peut la trouver par exemple dans l'Encyclopédie ou dans le Dictionnaire de musique de Rousseau, et qui est encore valable au XIX" siècle : " espèce de petit poème lyrique fort court, qui roule ordinairement sur des sujets agréables, auquel on ajoute un air pour être chanté dans les occasions familières $»$ (Rousseau, J.-J., 1977 [1767] : 115). Par métonymie, " l'air » est devenu la « chanson », et c'est sous ce vocable « d'air basque » que 
paraît le premier chant basque dont la musique ait été gravée, si l'on en croit J. Vinson (1884:20), qui situe cette publication vers 1820 . Cette date peut être précisée à l'examen de la partition, qui porte une vignette gravée en 1824. Gustave Dugazon (fils de la mezzo-soprano Louise Dugazon, compositeur d'opérascomiques, de ballets et de nombreuses romances, décédé en 1826) est l'auteur de l'arrangement à trois voix ad libitum - sur un texte, sans rapport avec l'original basque mais faisant allusion à l'Adour et aux montagnes, dû au baron de LamotheLangon - avec accompagnement de piano ou de harpe, de cet « air si connu dans le Labourd " (id.) : Epher zango gorri ${ }^{20}$.

\section{" Tous chantent " et certains improvisent}

À cette date cependant, on ne trouve pas de comptes rendus de concerts incluant des « airs basques » chantés. Le chant est néanmoins très répandu comme pratique quotidienne au Pays Basque ; Victor Hugo le souligne en 1843 : «à Pasages, on travaille, on danse et on chante. Quelques uns travaillent, beaucoup dansent, tous chantent "; « un matelot bayonnais chante du matin au soir » sous son balcon, les pêcheurs « tirent leurs filets de l'eau en chantant » (1984 [1843]: 91, 82, 83). Hugo mentionne le chant à plusieurs reprises, mais ne le décrit jamais, et ne fait aucun commentaire à son sujet. Il ne cherche pas non plus à apprendre des chansons mais par contre s'essaie à parler basque.

Jean Haritschelhar, dans son étude consacrée au poète souletin Etchahun (17861862), rappelle dans un chapitre «Musique et poésie » (1969:379-413) les liens multiples qui les relient en Pays Basque, et attestent de l'importance de la pratique vocale : "toute poésie populaire est chantée » depuis B. Dechepare au moins si l'on en croit la préface de ses Primitice (1545). Très fréquemment, le tex te même d'une chanson mentionne le fait qu'il sera chanté, et J. Haritschelhar insiste sur une strophe d'Etchahun définissant «l'idéal masculin des jeunes filles de la première moitié du XIX siècle $"$ : riche, mais aussi khantari et dantzari, c'est-à-dire bons chanteur et danseur. Quant au poème, il prend en basque le nom de « chant »kantu, kanta ou khantore, selon les dialectes: l'inverse de la démarche française qui voit dans la « chanson » une « poésie populaire».

Jean-Baptiste Orpustan, quant à lui, estime que la pratique poétique, « probablement intense dans le cadre de l'improvisation ou semi-improvisation chantée " entre 1650 et 1800 (1996:101), ne nous est parvenue que de façon marginale et souvent tardive, comme dans le recueil dit « de 1798 » : conservé au Musée Basque de Bayonne, il contient des chants allant de l'époque de Louis XIV à 1802, généralement anonymes, avec référence à l'air pour chacun et partie notée, dont les textes ont été publiés par. Patri Urkizu en 1987.

Mais revenons à une perspective historique. A. Mazure (1839:518) évoque lui aussi « le goût du chant si prononcé dans le Pays Basque », et enchaîne avec les "poètes improvisateurs ». Après la danse et la pelote, l'improvisation est en effet la spécificité basque qui commence à être relevée, à partir des années 1830 . On peut ainsi en trouver la mention à l'article «Basques » de l'Encyclopédie des gens 
du monde (de Walckenaer 1834), l'Encyclopédie catholique (M, J. de, 1842), le Dictionnaire de la conversation (Garay de Monglave, E., édition de 1873 : pas mentionné en 1833), et chez certains auteurs : C. Fauriel (1836:525), A. Chaho (1979 [1836] : 126), A. Mazure (voir plus haut), Francisque-Michel (1857 : 214), etc. Cette pratique témoigne donc aussi de l'habitude du chant parmi la population, avec laquelle elle est parfois confondue. Or si tout le monde chante, tous n'improvisent pas, et une chanson peut avoir une autre source que l'improvisation.

Pour A. Chaho cependant, là est bien l'origine du répertoire populaire chanté : les « chanteurs montagnards » improvisent sur « les drames de la vie politique et ceux de la vie intime », se constituant ainsi « une gazette toute en chansons », chansons qui « après être restées gravées quelque temps dans des mémoires privilégiées, se perdent sans retour au bout d'un petit nombre de générations » (Chaho, A., 1844 : «Chants basques »). Il faut donc intervenir pour les sauver de l'oubli. Comme Iztueta pour la danse, Chaho se montre alors un précurseur dans le domaine du chant basque, puisque c'est le premier qui envisage la publication d'un recueil (vingt-cinq ans avant la première publication effective), " pour dérober à ce grand naufrage ce qui nous reste encore de nos chants nationaux et de notre mélodie primitive ». Il envisage ce corpus comme « un objet de comparaison et un monument où respirera le génie poétique et musical de l'une des plus antiques peuplades de l'Europe » (op. cit.).

Ce recueil aurait eu pour titre, selon un prospectus paru dans le Tribly ([s. n], 1844 : [sans titre]), Chants basques, Romances, Mélodies, Chants de genre, Chansons de table, Récitatifs, Danses, Mélopées, etc. Ailleurs, A. Chaho annonce la parution des Chants populaires de la Navarre et des provinces basques (1845, $\mathrm{n}^{\circ} 50:$ : Poésie cantabre »). Il en publiera à plusieurs reprises des extraits (textes seuls) dans l'Ariel, sous l'intitulé de «poésies cantabres » en 1845. Mais la publication intégrale (soixante-dix pièces environ) n'a jamais eu lieu. Elle prévoyait de donner " les textes, recueillis de longue main et soigneusement épurés», auxquels seraient joints « leurs timbres, notés avec l'exactitude que réclame leur simplicité vénérable » (Chaho, A., 1844: "Chants basques »). «Les accompagnements de piano et de guitare sont confiés à des compositeurs de talent et de réputation " ajoute Chaho, sans préciser leurs noms. En fait de parutions, il se contentera d'orthographier les chansons basques publiées par P. Lamazou, qui est le premier à les porter avec efficacité sur la scène musicale, par le concert et par l'édition ${ }^{21}$.

\section{Lamazou, "écho fidèle " des " bons airs béarnais et basques "}

C'est en effet à un Béarnais, fils d'un tisserand, né à Pau en 1816, qui a été au Conservatoire de Paris l'élève de David Banderalli (professeur réputé et futur beaupère du compositeur bayonnais Adrien Barthe) que l'on doit la " publicité » du chant basque, sur le plan musical. Et c'est le public (grand) bourgeois de Paris qui le découvre en premier. Pascal Lamazou vit de leçons particulières, de concerts et de prestations privées données dans les salons de la capitale. À côté du répertoire lyrique habituel, il s'est fait une spécialité des « chants pyrénéens ", notamment lors de son concert annuel, qu'il donne très régulièrement à partir de 1846 et pendant trente ans à la salle Pleyel, au début du printemps. 
«Chants pyrénéens » est le terme employé dans la presse (Blanchard, H., 1853 : 161, par exemple), qui parle également de " mélodies béarnaises ", d" « airs basques » et plus généralement d' " airs de son pays », et. c"est aussi le titre du recueil que P. Lamazou fait paraître en 1869, comprenant trente-six. « airs béarnais », douze « airs basques » et deux « airs des Pyrénées orientales ». Pour la première fois (mis à part le recueil de J.I. de Iztueta, bien sûr, qui n'aborde les chants que par l'intermédiaire des danses chantées) se trouvent publiées conjointement paroles et musique. Ce recueil connaît assez rapidement deux autres éditions (1874 et 1877), qui y joignent une « légende préface » de Paul Lacôme ${ }^{22}$, une gravure de Gustave Doré, une opinion de Théophile Gautier parue dans le Journal Officiel et une lettre de Frédéric Mistral.

Après Paris, P. Lamazou chante à Bayonne « ces bons airs basques et béarnais dont il est né l'écho fidèle, ces fraîches mélodies pyrénéennes dont il est le vivant chalumeau » (A.B., 1854 : «Lamazou à Bayonne »). En 1856, on lui demande de venir donner, à la fête d'Urrugne, devant le prince Bonaparte, « les airs basques originaux si bien chantés par lui, et que les salons parisiens ont tant de fois applaudis » ([s. n], Courrier de Bayonne, 1856 : [sans titre]). La consécration parisienne fait-elle entendre d'une autre oreille le répertoire autochtone ? Les critères de jugement ont définitivement changé en tout cas : P. Lacôme (Légende préface, 1869) loue « la fraîcheur exquise, la grâce ingénue, l'originalité piquante des mélodies », accompagnant « le charme des poèmes » et leurs « couleurs locales très vives et très accentuées ». Le « chant pyrénéen », paroles et musique, est reconnu et apprécié. Néanmoins, on peut penser qu'il n'a pas encore atteint une reconnaissance complète : sa publication s'adresse, selon Lacôme, aux " curieux de toute taille". Faut-il donc être dans une disposition d'esprit particulière pour s'y intéresser ?

La publication de P. Lamazou n'est cependant pas la seule, ni même vraiment la première. Dans les années 1860 , on voit en effet se multiplier les témoignages d'intérêt pour une collecte des chants basques : « un organiste de Saint-Sébastien, M. San-Esteban [sic], s'occupe en ce moment de recueillir les airs nationaux des pays basques afin de faire connaître ces airs à la fois originaux et gracieux. Déjà quelques-uns de ces morceaux ont été publiés à Paris ", annonce Le Courrier de Bayonne ([s. n], 1862 : «Espagne »). Effectivement, le catalogue de la Bibliothèque Nationale de Paris rassemble un certain nombre de chansons basques parues en feuiliets séparés entre $\mathbf{1} 862$ et 1864 , sans nom d'auteur, dont les titres se retrouvent dans la Colección de aires vascongados que J.A. Santesteban lancera à SaintSébastien (sans doute à partir de 1864) ${ }^{23}$, mais pas forcément dans la même tonalité ni avec le même accompagnement de piano. D'autre part, on peut lire dans le Dictionnaire universel Larousse ([s. n]., 1867 : article « Basques ») : «à côté de la littérature écrite, qui est si pauvre, les Basques possèdent une autre littérature populaire, consistant en romances, en chansons, en ballades, qui ont été transmises. par tradition, et que conserve religieusement la mémoire des chanteurs. Malheureusement, ce côté original de la littérature basque ne nous est que fort imparfaitement connu, parce qu'on n'a pas encore rassemblé ces morceaux épars. Cependant, il en existe un recueil composé par $\mathbf{M}$. de Latena, mais qui est encore inédit ». De même J. Vinson signale-t-il « un lot de papiers, dont [il s'est] rendu 
acquéreur à Bayonne, dans une vente publique » : il y a trouvé « beaucoup de chansons basques et beaucoup de musique : la plupart de ces papiers venaient de [son] ami regretté Alexandre Dihinx, les autres avaient appartenu au brillant, mais dangereux, écrivain basque Augustin Chaho » (1883 : XV).

A côté de ces collectages restés confidentiels, les premiers recueils paraissent à la fin du Second Empire, sous la forme habituelle à l'époque : notation solfégique, texte basque et traduction ou adaptation française ${ }^{24}$, accompagnement de piano réalisé par un musicien muni du bagage technique nécessaire ou un compositeur - si possible (re)connu, de sorte que sa participation parachève le changement d'état du chant populaire harmonisé. On relève par exemple, pour les Cinquante chants pyrénéens, recueillis, chantés et publiés par Pascal Lamazou, 1869 : D.F.E. Auber - alors directeur du Conservatoire de Paris, C. Gounod, A. Barthe - Grand Prix de Rome, originaire de Bayonne, F. David, J.B.Weckerlin - compositeur et folkloriste, le jeune Massenet, etc. L'accompagnement opère véritablement une transformation du statut de la mélodie populaire, « la prenant simple fille des champs et l'introduisant dans les salons, de par l'imprescriptible autorité du bon goût ", comme le dit P. Lacôme à propos de Lamazou. Il s'agit d"une sorte d'élévation dans l'échelle sociale, c'est-à-dire dans la hiérarchie des genres, car l'accompagnement « relève » (Lacôme), « rehausse » (Gautier) « ces petites merveilles de grâce et de sentirnent » (Lacôme), « ces mélodies exquises » (Gautier). Ainsi parée, pliée aux normes strictes de la musique savante (de la notation à l'interprétation), la « muse populaire » (Lacôme) peut donc sortir dans le monde, et ses productions, échappant à l'oubli qui les guette lorsqu'elles restent dans le donaine populaire, peuvent prétendre à la longévité, voire à l'éternité lorsqu'elles ont été «fixées pour toujours sous les auspices des plus illustres maîtres »(Mistral).

\section{" La grâce rustique et naïve " des Airs basques}

Publiés à Paris en 1869 également, les Douze airs basques choisis et notés par $M^{m e}$ de la Villehélio - et dotés par ses soins d'un accompagnement de piano - cherchent moins peut-être à se plier aux canons de la musique de salon contemporaine : ce que l'auteur (Julie Adrienne Carricaburu, dite Hortense, née au château de Chéraute en 1827 et élevée au Collège du Sacré-Cœur) valorise et voudrait transmettre, c'est leur «grand charme et [leur] frappante originalité ». Est-ce l'influence d'A. Chaho, qui fut son précepteur pendant les vacances? En tout cas, Madame de la Villehélio s'est montrée plus sensible que P. Lamazou à la façon dont ces 《 airs », auxquels elle se serait intéressé dès 1848-50, lui ont été chantés. Dans d'intéressantes « Observations préliminaires », elle signale la présence de quart de tons, « et probablement de fractions moindres, qu'il est difficile de préciser » ; l'habitude du chanteur de commencer par " une sorte de grupetto intraduisible ", " sorte de cadence [qui] se rapproche de celle des airs des vieux maîtres des XVII" et XVII" siècles Couperin, Rameau, etc. », et de finir par un long point d'orgue qui lui paraît être le signe de ce que « le chanteur habite la montagne et qu'il attend la réponse de l'écho »... Pour ses accompagnements, elle a cherché à reproduire l'effet des instruments traditionnels (le « tambour à grelots », le « flageolet », une 
" sorte de boîte longue » dont la description est celle du ttun-ttun), « ne voulant user $[\ldots]$ que des ressources musicales du pays, y ajouter au-delà d' une certaine mesure [lui] semblerait compromettre la grâce rustique et naïve de ces chants ".

En ce qui concerne les chants eux-mênes, Madame de la Villehélio les présente comme « de vieux airs, dont quelques-uns d'après leur facture remonterait à l'époque des chants grégoriens », de forme simple, échappant à la tonalité moderne « en raison de ce que [les Basques] ont toujours vécu en dehors de toute assimilation avec leurs voisins », et elle en profite pour dresser un portrait de ses compatriotes hérité des clichés en cours depuis le début du siècle (" peuple primitif aussi vieux que le monde ", à l'origine mystérieuse, animé de passions extrêmes et non policées) et propre à expliquer les caractéristiques musicales qu'elle leur trouve. Il émane de ses propos, rendus très personnels par l'emploi de la. première personne du pluriel (une manière de « nous, les Basques »), une certaine volonté de donner à des non Basques des éléments pour mieux appréhender les productions musicales de cette «épave de l'Antiquité 》-et elle ira jusqu'à leur conseiller des lectures (Francisque-Michel, Chaho, le vicomte de Belzunce) qui leur feront «passer quelques heures charmantes ».C'est le côté impénétrable, mystérieux des Basques qui est mis en avant, après avoir présenté l'intérêt de leurs «vieux airs ».

Pas de préface, en revanche, pour l'ouvrage paru en 1870 - et dédié « au Pays Basque aimé » - de l'avocat J.D.J. Sallaberry (souletin comme Madame de la Villehélio, et de dix ans son cadet), mais quelques observations sur l'orthographe de la langue basque, et en fin de volume, des notes sur les chants, égratignant parfois au passage le travail de P. Lamazou. Alphonse Dotterer, « jeune musicien d'avenir ${ }^{25}$, ancien élève du Conservatoire de Paris », a harmonisé quinze de ces chants.

\section{Des instructions peu respectées}

Cette façon de procéder n'est pourtant pas celle qu'ont essayé d'imposer les Instructions pour un Recueil général de poésies populaires de la France auxquelles nous avons déjà fait allusion. Diffusées en 1853 sous l'égide du ministère de l'Instruction publique et des Cultes, elles se proposent de « déterminer le véritable caractère des chansons populaires » (Cheyronnaud, J., $1997: 56$ ) et donnent des instructions quant à leur collecte. On y voit bien le renversement qui s'opère sous le Second. Empire, où la musique tend à devenir l'égale de la poésie, sinon à occuper la première place devant le texte qui, jusque là, monopolisait l'attention. « La mélodie joue incontestablement un rôle considérable et quelques fois même le rôle principal dans les chants populaires ; il n'est point de chant populaire proprement dit sans mélodie ", estime Edmond de Coussemaker lors des travaux préparatoires aux Instructions (op. cit : 74).

Celles-ci précisent deux règles, nouvelles par rapport à l'attitude généralement adoptée jusque là, mais incontournables aux yeux du comité rédacteur : d'une part, ne pas chercher à débarrasser les mélodies entendues de " cette rouille précieuse » 
que représentent certaines caractéristiques particulières ayant trait à la tonalité et à l'irrégularité du rythme, car « ces caractères [...] sont pour elle comme un cachet d'antiquité ", et d'autre part, ne pas composer d'accompagnements. Bref, "écrivez l'air tel que vous l'entendez chanter, et ne changez rien " (ibid. : 94-95). Telle n'était pas, on l'a vu, l'attitude des premiers collecteurs basques - Madame de la Villehélio étant sans doute la plus proche des Instructions dans sa démarche sinon dans sa publication.

Mais quels sont les résultats de l'enquête Fortoul en ce qui concerne le Pays Basque ? Plusieurs informateurs sont mentionnés, Hippolyte Durand à Bayonne et l'abbé Théodore Laran à Larressore, qui ne semblent pas avoir été très efficaces, et trois autres personnages, qui ne portent pas le titre de « correspondants » départementaux comme les précédents : Eugène Garay de Monglave, à la «probité littéraire fort douteuse » (J. Vinson $1884: 49$ ) qui $n$ 'hésite pas à adresser des chants de sa fabrication ou recopiés dans des revues pyrénéennes ou bayonnaises (onze au total, d'après J. Vinson $1884: 55$ ), l'abbé Fourcade, inspecteur primaire de l'arrondissement de Bayonne, dont aucun chant populaire n'est retenu, et un Souletin inspecteur primaire de l'arrondissement de La Réole (Gironde), Jean-Baptiste Archu, qui adresse une douzaine de pièces « comme spécimen d'une collection de cent vingt chants populaires du pays basque qu'il a rassemblés » (Cheyronnaud, J., 1997: 70).

Dans les Instructions elles-mêmes, deux chants sont donnés (en traduction. française, selon la règle choisie par le comité) : des fragments du fameux Chant de Lelo, et « une chanson dont la traduction a été dictée à $\mathrm{M}$. de Quatrefages par une vieille femme de Biarritz ", intitulée Santa-Clara (op. cit. : 88-89). Maigre bilan comparé au millier de pièces rassemblées, pour une région qui s'enorgueillit aujourd'hui de son patrimoine vocal... En fait, le temps de la collecte n'est pas encore venu pour le Pays Basque, contrairement à d'autres régions qui auront été explorées bien plus tôt que celui-ci. Rappelons, en dehors même de l'enquête Fortoul, les recherches dont a fait l'objet la Bretagne à partir de la fondation de l'Académie celtique en 1805 , et le retentissement de la publication, en 1839, du Barzaz Breiz de Th. Hersart de la Villemarqué.

\section{"Chants pyrénéens »}

Revenons aux premières publications concernant le Pays Basque. Ce n'est pas la caractéristique d'être basque ou le rapport avec le Pays Basque qui sont mis en avant par leur titre, mais le lien avec la thématique du " pyrénéisme » : après les Chants pyrénéens de P. Lamazou, les Douze airs basques de Madame de la Villehélio portent le titre de Souvenirs des Pyrénées ${ }^{26}$. Dans le même ordre d'idées, Francisque-Michel avait joint à sa courte étude de la musique basque trois airs de danse empruntés à Iztueta (dont un avec paroles) et une " cantilène » qui lui semble représentative des « chansons de nos montagnards » (1857: 435). Le Pays Basque " n'existe " pas encore pour le public bourgeois, alors que la montagne (Alpes et Pyrénées), révélée par les Romantiques, est devenue un thème littéraire et pictural, et un lieu de villégiature touristique et thermale. On peut peut-être penser aussi. 
que ces recueils " pyrénéens » s'adressent plutôt au public urbain, parisien en premier lieu, et que le rapprochement Pays Basque-Béarn/Pyrénées lui permet de situer géographiquement ces régions ; inversement, J.D.J. Sallaberry fait paraître en 1870 à Bayonne ses Chants populaires du Pays Basque où les Pyrénées ne sont plus mentionnées. C'est donc une situation topographique et une association avec un lieu repéré et connoté qui identifie le chant basque : il n'est pas encore porteur d'une image assez forte et précise pour être présenté en tant que tel.

Peut-être aussi la dénomination de « chants pyrénéens » pour les chants popülaires basques et béarnais prend-elle la suite des publications de mélodies et pièces instrumentales de musique savante qui ont elles-mêmes exploité le filon pyrénéen à la génération précédente (Souvenirs des Pyrénées édités par Philippe Musard, 1841 , Souvenir des Pyrénées, op. 13 de D. Alard, 1844, Souvenir des Pyrénées de Désiré Magnus, 1850, Le boléro des Pyrénées ou les souvenirs de la Navarre d'Alfred Roland, s.d., Les Pyrénées, souvenirs des montagnes d'Adrien Louis Boïeldieu, 1856, par exemple), voire deux générations plus tôt (l'air basque utilisé par G. Dugazon et mentionné plus haut est publié également sous le titre de Souvenirs des Pyrénées [sic]). Le Souvenir est d'ailleurs un genre qui se maintient, s'étendant au Pays Basque « découvert » par Napoléon III et sa femme. Il représente encore un cas de pièce instrumentale basée sur des mélodies populaires puisque J. Vinson donne (1884:23), comme premiers arrangements connus de chants basques, un Souvenir de Biarritz, quadrille basque d'Alexandre Artus (1860) où figurent Adios, ene maitea et Chapel goria [sic], et des Souvenirs du Pays Basque pour piano de Claude Déplace [vers 1863], recueil de valses intitulé Lore eskualdun ahurra et contenant une vingtaine de chants basques encore parmi les plus célèbres aujourd'hui'27.

Le " chant basque " est aujourd'hui une notion plus reconnue que le « chant béarnais ». Or il est intéressant de noter que la situation était toute autre dans la première moitié du XIX $X^{*}$ siècle : le Béarn connaît avant le Pays Basque la publication de « chants nationaux béarnais » (Emile Vignancour, 1827 et 1852 ; Xavier Navarrot, 1834 ; Frédéric Rivarès : Chansons et airs populaires du Béarn, 1844) ${ }^{28}$, sans parler des Noëls béarnais, dont certains sont publiés dès 1756 par Henri d'Andichon. L'Essai sur la musique ancienne et moderne de Jean-Benjamin de Laborde donnait en 1780 des exemples (paroles et musique) de « chansons béarnoises » (Laborde, J.-B., 1780 : 152-156). Cet intérêt est sans doute apparu grâce au personnage de Cyprien Despourrins (né en 1698), poète et auteur de chansons éditées en 1820 (Estrées béarnaises), à qui un monument est élevé en 1840 dans son village natal d'Accous, et à celui du ténor Pierre Jélyotte, célèbre pour avoir chanté à la cour de Versailles des airs béarnais (bien avant que Garat ne chante ses romances à Marie-Antoinette, puisqu'il s'adressait à Louis XV). Enfin, rappelons que c'est P. Lamazou qui porte les chants pyrénéens sur la scène de concert, suivant les traces « d'un ténor de l'Opéra né à Pau, Lavigne, qui fit beaucoup parler de lui au début de la Restauration » : celui-ci chanta avec succès une chanson. de Despourrins sur la scène de plusieurs théâtres (Bénichou, P., 1970: 116). Les recueils béarnais, même s'ils sont « du félibrige avant la lettre, et non de la poésie orale » (op. cit. : 115) sont souvent cités et commentés (P. Benichou parle par exemple d'un article du Globe le 28 avril 1829). 
Pour en terminer avec le pyrénéisme, revenons rapidement sur le terme de «montagnard » et précisons deux points : tout d'abord, l'association Basques/montagnards est ancienne et très répandue au XIX ${ }^{\circ}$ siècle. Parmi toutes les étymologies fantaisistes circulant au début de ce siècle, l'une d'elles fait même découler le mot « basque » de «basac-hoc, bascos, peuples sauvages, montagnards [sic] " (Garay" de Monglave, E., 1833 : article « Basques »). On considère que l'influence de la montagne est double : d'une part, elle protège ou a protégé les Basques (des envahisseurs comme des influences extérieures), et d'autre part, elle « déteint » et donne ses caractéristiques au chant. Dès 1818, Etienne de Jouy (par ailleurs librettiste de Spontini et Rossini) formule une appréciation qui fera florès à la fin du siècle : « les chants des Basques sont langoureux comme dans tous les pays de montagne où le séjour des hommes, dans ces hautes régions terrestres, semble disposer leur âme aux sensations les plus tendres » (1818: 152). Même au milieu des collines ou au bord de la mer, les Basques sont donc « les montagnards des Pyrénées occidentales » (C. Fauriel), comme à Urrugne où, en 1853, la poésie primée aux fêtes basques est chantée par « un chœur nombreux de montagnards » ([s. n], Le Courrier de Bayonne, 1853 : " Fêtes d'Urrugne ").

Ceci nous conduit à une seconde précision, qui concerne le chœur de quarante voix d'hommes organisé en 1832 à Bagnères-de-Bigorre par Alfred Roland, chantant un répertoire composé par leur chef et exaltant sous toutes ses formes le thème de la montagne (Mabru, L., [s.d.] : 48) : les fameux Montagnards, qui parcourent l'Europe et le Proche-Orient de 1838 à 1854, ont sans doute contribué eux aussi à cette association Pyrénées/chant (populaire par les interprètes, sinon par le répertoire). Ont-ils influencé la pratique chorale en Pays Basque ? Leur passage à Bayonne en 1837 suscite certes une certaine émulation: Le Phare de Bayonne parle de «nos chanteurs bayonnais [de la Société Philarmonique, placés sous la direction de Masson, un élève d'A. Choron], maintenant engagés avec tant de chances de succès dans la lutte avec les montagnards de Bagnères » (Stein, L., 1837 : "Concert de la Société Philharmonique »). Comme eux, ils terminent leurs prestations par « un chant de montagne », La haüt sus las mountagnes de Despourrins (Y., 1837 : « Concert de la Société Philarmonique »). Mais par la suite, il est difficile de trouver des signes d'une influence certaine : la chorale de la Société Philarmonique disparaît au bout de quelques années, et on ne relève pas par la suite de chœur basque constitué à l'exemple des Montagnards de Bagnères-de-Bígorre. Dans les années 1860, des orphéons (dont l'histoire en Pays Basque reste: à faire) apparaissent dans les villes (avec un répertoire de musique savante typique de l'institution orphéonique), et même dans les grosses agglomérations et les villages (Saint-Jean-de-Luz, Saint-Palais, Sare par exemple : avec un répertoire peut-être plus axé sur le chant basque, mais très mal connu).

Il semble qu'A. Roland se soit aussi intéressé aux interprètes basques, puisque "le double quatuor des huit artistes-basques » (un otxote avant la lettre ?) donne le 29 septembre 1856, devant «LL. MM. l'Empereur et l'Impératrice des Français et de toute la Cour » en villégiature à Biarritz, une audition du Premier recueil des chants favoris de celui-ci (si l'on en croit le titre de la publication déposée à la Bibliothèque Nationale). Curieusement, la presse locale ne fait aucune mention d'une pareille prestation à cette date. 


\section{"Chant basque " et goût impérial}

Malgré la vogue du pyrénéisme, on constate que l'usage du terme « chant basque » est devenu courant dans la presse bayonnaise des années 1860 ; il ne fait plus référence uniquement aux chants historiques, mais plutôt à ce que nous appelons aujourd'hui chants populaires, et l'aspect musical a été considérablement revalorisé - première étape d"une évolution qui amènera à laisser tomber le texte. L'incompréhension de celui-ci, puisque les chants sont donnés en basque, ne pose apparemment pas de problème.

C'est ainsi que nous apprenons que Napoléon III et Eugénie de Montijo sont de grands amateurs de « chant basque » : parce qu'il a été mis à la mode (ou du moins est devenu objet d'écoute musicale, grâce à P. Lamazou en particulier), mais aussi parce que Napoléon III tient à encourager une pratique rurale et non séditieuse, par opposition aux pratiques urbaines plus subversives? Dans le rapport d'H. Fortoul au sujet du Recueil des poésies populaires de la France (Cheyronnaud, J., 1997 : 51), il y est rappelé que le Prince Président est le « fondateur d'un gouvernement qui aime à s'appuyer sur la fidélité des souvenirs poétiques du peuple ».

Lors de ses déplacements et randonnées en Pays Basque, le couple impérial en réclame fréquemment. Ainsi Eugénie, après avoir entendu le « chant basque » Donostiaco hirur damacho [sic] interprété par les orphéonistes de Saint-Jean-deLuz en 1865 , le demande à $M$. Doyambéhère, le curé, qui lui adresse «paroles basques, traduction française et musique, rien n'y manquait »-d'après Le Courrier de Bayonne (D., 1865 : [sans titre]). Ou encore : [à Saint-Jean-de-Luz toujours] «Sa Majesté a demandé des chants basques, mais l'orphéon étant dissous, il s'est écoulé quielques instants avant qu'on pût réunir les chanteurs qui en faisaient partie. On a cependant réussi à former un chœur qui a été rendu avec beaucoup de goût : Ay Madelen et le Caïcoua, chants favoris des provinces basques ", ([s. n], Le Courrier de Bayonne, 1867, $\mathrm{n}^{\circ} 2245$ : [sans titre]).

À Sare, le contrebandier Michel Dihursubéhère reçoit l'empereur et ses amis, et organise des prestations à l'intérieur des grottes voisines de Zugarramurdi : la grotte est « ouverte aux deux extrémités et surmontée d'une voûte d'une longueur considérable. Là, les voyageurs ont invité leurs guides à entonner quelques chants du pays, et tout à coup des voix magnifiques se sont fait entendre avec un admirable ensemble et ont fait retentir de leurs vigoureux et harmonieux accents les échos de ces lieux qui semblaient voués à un éternel silence » ([s. n], Le Courrier de Bayonne, 1866 : [sans titre]). Prosper Mérimée raconte lui aussi l'expédition dans l'une de ses lettres, précisant que la « douzaine d'orphéonistes ont chanté en chœur accompagnés par une espèce de flageolet très aigu, des airs basques d'un caractère très original » (Jean-Pierre, H., $1965: 422$ ).

De même, l'année suivante : «les guides, qui font partie de l'Orphéon de Sare, se sont alors écartés [après avoir servi une collation] et, se réunissant dans l'intérieur de la grotte, ils ont chanté divers airs basques et entr'autres [sic] les compositions couronnées au dernier concours de poésie. Le chant était large et puissant ; la sonorité de ces voix mâles, redoublée par l'écho, produisait un effet saisissant. 
L'Empereur a complimenté les chanteurs et, sur le désir qu'il a daigné manifester de les entendre de nouveau, nos virtuoses montagnards se sont empressé d'accéder à cet auguste vœu » ([s. n], Le Courrier de Bayonne, 1867, n 2246 : [sans titre]). On voit donc que l'aspect musical des œuvres et surtout la façon de chanter sont pris en compte. De plus, ce chant basque est une pratique collective, populaire et masculine (alors que l'air basque avec accompagnement de piano était le fait d'un seul exécutant, homme ou femme, à la technique de chant savante). On commence à le percevoir en tant que forme festive privilégiée et caractéristique des Basques.

\section{Charles Bordes et les Archives de la Tradition Basque}

Cette évolution dans l'image, la conception et le statut du « chant basque », qui s'affirme dans la deuxième décennie de l'Empire, prépare une diffusion plus "grand public » à la génération suivante. Un musicien tourangeau, Charles Bordes, a joué un rôle important dans cette diffusion à partir de la fin du siècle. En 1885, il assiste à une conférence de Julien Tiersot sur la chanson populaire, accompagnée d'auditions de pièces « révélées pour la première fois en ce jour » (Tiersot, J., 1909 : 21). À l'audition ( « en extase ») de Chorinoak kaiolan, « ce fut pour lui conme la suggestion d'une musique inconnue, sortie de l'autre monde » (id.). En 1889-90, il est envoyé en mission au Pays Basque par le Ministère de l'Instruction publique pour recueillir des chants populaires, et il y passe ensuite tous ses étés, devenant selon J. Tiersot « une sorte de Basque d'adoption » (id.).

Ces chants paraissent à Paris dans le cadre des Árchives de la Tradition Basque, qui se définissent comme des " documents pour servir à l'histoire du peuple basque " publiés " par un groupe d'écrivains et d'artistes » : la première parution annoncée est celle des Cent chansons populaires basques, recueillies et notées au cours de sa mission par Charles Bordes ${ }^{29}$, qui inaugurent une nouvelle étape dans la collecte. Dorénavant, ce sont des compositeurs qui vont sur le terrain et qui font ensuite connaître le fruit de leur recherche, en notation traditionnelle (ou en notation carrée), avec ou sans accompagnement musical, avec le texte basque et une traduction éventuelle, mais volontiers accompagné de commentaires ou de conférences explicatives.

Ainsi la conférence faite dans le cadre du Congrès de la Tradition basque tenu à Saint-Jean-de-Luz en 1897 et publiée deux ans plus tard, avec 54 mélodies notées, est-elle particulièrement importante. Charles Bordes y « démontre » l'intérêt musical du " chant basque », en l'analysant pour la première fois sur la base de critères musicologiques; la parenté qu'il lui trouve avec le plain-chant et l'aspect rythmique retiennent son attention. le chant basque s'inscrit maintenant dans une histoire musicale ${ }^{30}$, et non plus seulement dans une histoire littéraire, et des passerelles sont établies avec « la musique artistique » (Bordes, C., $1899: 307$ ), ce qui lui permet de comprendre (et donc de savourer) « le vague et la bizarrerie » qui troublaient tant les George Amé du milieu du siècle. Dans cette optique surgit alors un paradoxe que les collecteurs expriment de façon plus ou moins explicite : il faut être savant pour goûter la musique populaire - et le paysan qui chante «n'a pas la cul- 
ture dont peut-être on a besoin pour savoir apprécier la beauté originale d'une mélodie rustique ", estime le Père Donostia, musicologue (1923 : 685)... La conférence de Bordes aura son pendant à Bilbao en 1901, avec celle de l'ethnologue, linguiste et compositeur Resurrección María de Azkue, intitulée La música popular baskongada et accompagnée de 14 illustrations musicales (en attendant celles du Père Donostia, publiées en 1917 avec leurs 31 exemples musicaux\}.

Les mélodies recueillies sont intégrées à la composition d'œuvres de musique: savante (J. Tiersot y voit, dans le cas de Bordes, la volonté de « tirer des idées qu'il préconisait leurs plus multiples conséquences », op. cit. : 26) : signe que la musique a définitivement conquis la première place devant le texte, et que l'on considère que la « quintessence basque » se trouve dans la partie musicale. De plus, la chanson basque apparaît cornme " très particulière ", " d'une complète nouveauté " (ibid. : 22) par rapport aux autres traditions folkloriques : cette originalité qu'on lui prête légitime le fait qu'elle commence à être perçue comme spécificité basque et qu'elle serve de support à des compositions musicales, dans tous les genres (Suite basque pour flûte et quatuor à cordes, ou Rapsodie basque pour piano et orchestre de C. Bordes, par exemple), mais en particulier dans l'opéra.

\section{" Un peuple qui chante »}

Le premier opéra se présentant comme « opéra basque » est créé en 1884 à Saint-Sébastien, et fait grand usage de chants bien connus dans cette ville, notamment des zortziko d'Iparraguirre - arrangés par José Antonio Santesteban, l'auteur de la collection d'Aires vascongados commencée en 1862 à laquelle on a fait allusion plus haut. Il apparaît d'ailleurs sur certaines annonces comme "ópera de aires vascongados ». Petit à petit, les ouvrages lyriques se mettent à utiliser des " chants populaires basques ", principe qui fait le succès de Maitena, d'Etienne Decrept et Charles Colin en 1909, où l'on peut reconnaître plusieurs mélodies présentes dans le recueil de J.D.J. Sallaberry. L'année suivante, trois autres opéras de cette veine sont créés à Bilbao et leur succès incite un député à proposer la création d'un prix d'au moins 5000 pesetas pour l'auteur de la meilleure collection d'airs basques, af in que les compositeurs puissent disposer d'un fond plus important. Le projet est accepté, et les résultats du concours connus en 1912 : R.M. de Azkue propose un ensemble de 1800 pièces inédites, et le Père Donostia plus de 500 .

C'est certainement pour beaucoup la révélation de la richesse du patrimoine basque en ce domaine, que Bordes avait laissé entrevoir - et la preuve matérielle que les Basques sont bien ce qui se dessine depuis un demi siècle : « un peuple qui chante " (titre d'un ouvrage de Jean Ithurriague paru en 1947). Alfredo de Echave, librettiste de plusieurs « opéras basques », l'avait exprimé en 1910: "l'intuition musicale est un don caractéristique de notre peuple, [peuple] chanteur par excellence ", estimait-il au vu du nombre de musiciens professionnels et de l'importance de la musique populaire en Pays Basque. Il parle de celle-ci comme d'un «trésor » et du travail d'Azkue comme d'un " véritable monument à notre tradition musicale » (1910:3-6). Cette idée est ancienne et avait été reformulée au début 
du XIX ${ }^{\circ}$ siècle, on l'a vu, mais elle ne se diffuse dans le grand public qu'un siècle plus tard. De plus, les chants historiques ne sont plus du tout la catégorie privilégiée : « au Pays Basque, la chanson légendaire ou héroïque, la complainte historique est tellement exceptionnelle qu'on peut dire qu'elle ne compte pas », estime par exemple Charles Bordes (1899: 309). La nomenclature proposée par R. M. de Azkue comprend des chansons amoureuses, bachiques, des berceuses, danses, complaintes et élégies, des épithalames, des chansons festives ou humoristiques, enfantines, narratives, de métiers, religieuses, des romances, des chants de quête, et quelques pièces épiques ou satiriques qui seront finalement dispersées dans d'autres rubriques (Azkue, R. M. de, 1990 [1922] : 54).

Parallèlement, de plus en plus de Basques deviennent chanteurs lyriques professionnels (en particulier des ténors, à la suite de Julián Gayarre, 1844-1890). De ce phénomène, Madame Bovary (1857) offrait déjà une illustration : le célèbre Edgar Lagardy, qui tient le rôle d'Edgardo dans la représentation de Lucia di Lammermoor à laquelle Emma Bovary assiste au théâtre de Rouen, a été découvert par une princesse polonaise qui, « l'écoutant un soir chanter sur la plage de Biarritz, oủ il radoubait des chaloupes, en était devenue amoureuse " (Flaubert, G., $1964: 650)^{31}$. La Baskonia écrit en 1896 ([s.n.], $1896: 24$ ) : « d'ici peu de temps, les ténors basques vont monopoliser la scène lyrique ". Mais, en fait, y a-t-il plus de chanteurs basques que béarnais par exemple ? N'est-ce pas un effet de l'association basque/chant qui induit à remarquer la présence (certaine) des Basques, alors qu'on ne notera pas ou moins la présence (certaine) des Béarnais ?

Notons aussi que le développement de l'opéra basque a été rendu possible par les chorales, qui se multiplient à cette époque. Il serait intéressant d'analyser les. conséquences de ce (double) phénomène sur la pratique du chant et sur la façon de chanter en Pays Basque. Leur répertoire comprend également des chants basques harmonisés pour voix seules ou avec accompagnement (qui restent, aujourd'hui encore, à la base du répertoire des chœurs dits ou se voulant " basques »). Ainsi le corpus recueilli, généralement en milieu rural, se diffuse-til en partie (pas forcément parmi les mêmes couches de la population) sous une autre forme, et passablement modifié - contribuant au passage à fixer les versions des airs qui rencontrent le plus de succès, comme cela sera plus tard le cas avec le disque et le chanteurs ou groupes reprenant des chants populaires.

Il faudrait aussi étudier le rôle des chorales (en particulier par leurs tournées hors du Pays Basque, à partir d'Eresoinka, formé à Sare avec les réfugiés fuyant le franquisme) pour la diffusion de la figure du « Basque chantant » à l'extérieur du pays. C'est l'un des clichés des années trente, période où l'apparition du tourisme de masse amène à la constitution de stéréotypes régionaux : chant collectif, danse et pelote illustrent - définissent - le Pays Basque à l'Exposition Internationale de 1937 à Paris. De même, on pourrait s'interroger sur l'impact, dans les années 50 , de chanteurs de musique légère (opérettes, comédies musicales, films musicaux), en particulier Luis Mariano, dans la diffusion française d'une certaine image du chant (eur), de la musique et du Pays Basque. 


\section{L'opéra, « chant populaire transformé "}

Le théâtre lyrique a apporté cependant au " chant basque » une dimension que l'ensemble vocal n'atteint pas (ou du moins pas autant). Jusque là, on l'a vu, la simplicité, la « rusticité » du chant basque, son origine populaire apparaissaient comme des caractéristiques négatives, l'empêchant d'accéder au statut d'œuvre d'art. Parlant en 1857 des « chansons basques », Francisque-Michel, par exemple, craignait « que le siècle actuel, habitué aux artifices de notre poésie et à l'éclat de notre musique moderne, ne trouve fades les naïfs accents de la muse des montagnes et les accords sans art de son pipeau rustique » (1857:221). Ces caractéristiques négatives deviennent peu à peu (pas seulement au Pays Basque, mais dans toute l'Europe, et tout particulièrement grâce au rayonnement de la Schola Cantorum, fondée par Vincent d'Indy, Alexandre Guilmant et Charles Bordes, où sont formés plusieurs compositeurs basques) des qualités recherchées, car on considère qu'elles permettent un renouvellement du langage musical, arrivé dans une impasse.

Mais la musique « populaire » n'est pas perçue pour autant comme égale, dans la hiérarchie des genres, à la musique savante : c'est le chant populaire (un certain chant populaire même ?) transformé par l'art savant qui a un véritable statut musical, et ce d'autant plus que la forme qui l'accueille est valorisée. Or l'opéra est encore, à la fin du XIX et au début du XX $X^{e}$ siècle, un «genre noble » au vu de sa complexité musicale et de son prestige social. Porter sur la scène lyrique le chant popülaire - jugé jusque là fruste et sans intérêt artistique c'est donc lui octroiyer une nouvelle valeur. Lorsqu'il se saisit de ce matériau et l'intègre à son ouvre, le compositeur d'opéra accomplit ce qui est considéré comme une façon de le signifier. Cela lui est d'autant plus aisé qu'on estime qu'il y a une continuité entre le chant traditionnel, expression riche, mais primitive et limitée, de la sensibilité populaire, et l'opéra, produit raffiné, complexe et développé, méritant pleinement le nom d'art. Felipe Pedrell, le maître à penser de beaucoup de compositeurs espagnols de cette période, l'a théorisé dans son célèbre ouvrage, traduit en français sous le titre Pour notre musique : " le drame lyrique national est le lied développé dans les proportions voulues par le drame ; c'est le chant populaire transformé » $(1893: 36)$.

\section{Une nationalité musicale}

L'utilisation du folklore musical, comme on l'appelle alors couramment, obéit également à l'idée largement répandue, à partir de la. fin du. XIX ${ }^{\circ}$ siècle, selon laquelle il est ĺa meilleure expression-et/car l'expression directe-des caractères physiques, psychologiques et moraux des populations, voire du pays, dont il émane. La conférence de C. Bordes, intitulée La musique populaire des Basques, commence par quelques considérations qui illustrent ce principe : «c'est surtout de l'art populaire, art d'intuition et d'originalité, qu'on a pu dire qu'il exprime fidèlement le caractère de la race, qu'il reflète avec clarté et profondeur les traits essentiels du pays où cette race a fixé sa vie. Cette loi, maintes fois vérifiée, s'applique d'une manière frappante à la musique basque. Rien ne fait mieux connaître 
un Basque que sa chanson. Elle traduit, dans sa langue naïve et charmante, les vives. sensations et les fiers sentiments qui composent cet être admirable. Nous y saisissons toute son humeur et toute son âme, ses qualités primesautières et traditionnelles, son ardeur, sa gaieté, son amour de liberté, sa joie de vivre et son mépris de: la mort, et surtout sa foi robuste, plus morale que mystique, qui donne au Labourdin tant de noblesse et de sérénité ». Plus loin, il ajoute : " cette musique n'exprime pas seulement les sentiments et les sensations du Basque, elle a encore une mystérieuse correspondance avec sa vie physique, son travail, son jeu ou sa danse [...]. Et le poète ira plus loin encore : il ne pourra entendre quelques-uris de ces thèmes, sans voir le Pays Basque lui-même, l'enivrante nature de ce coin de terre que Loti a su si bien peindre dans son roman de Ramuntcho » (Bordes, C., 1899 : 297-298).

En conséquence de quoi le « chant basque » apparaît comme le plus sûr moyen de conférer une nationalité (le terme est pris ici sans lui attribuer forcément une connotation politique) à la musique savante quil l'utilise (un opéra, une symphonie, seront basques s'ils font usage de «thèmes basques »). Une telle idée ne pouvait que rencontrer un accueil favorable auprès des nationalistes (on passe bien ici, en revanche, sur un terrain politique) dans le premier tiers du $\mathrm{xx}^{6}$ siècle, pour qui le chant basque apparaît comme l'illustration de l'essence immuable et éternelle du peuple basque et du pays : son emploi sera donc revendiqué pour l'expression et la défense de l'identité euskarienne. Une critique de l'opéra Mendi-Méndiyan lors de sa création à Saint-Sébastien en $19: 1$ l'exprime en un raccourci saisissant: «Et l'art basque descendit des montagnes » ([s. n], Euskalerriaren Alde, 1911 : 227).

En un siècle, on a donc fait passer le « chant basque » d'un texte poétique, à contenu historique, à une mélodie «nationale », expression sonore d'une identité basque. Curieusement, un « chant basque " parmi les plus connus rassemble en quelque sorte ces deux aspects : le Gernikako arbola de José María Iparraguirre, chanté pour la première fois en 1853 dans un café madrilène, rappelle les fors dont bénéficiaient les Basques et devient très rapidement populaire, jouant le rôle d'un véritable hymne national des deux côtés de la frontière. La mélodie n'est pas d'Iparraguirre comme on le croit généralement, mais consiste en une adaptation d'une danse biscayenne entendue probablement en 1836 à Durango (Arana Martija, J.A., $1982: 127$ ).

Le personnage d'Iparraguirre lui-même est à la croisée des domaines savant: et populaire : ce « barde » (suivant la qualification romantique qu.'on lui donne très tôt), auteur de quelques chansons «plus remarquable [s] par la vigueur sonore et le dynamisme de [leurs] textes conçus en même temps que l'air pour les chanter que par leurs qualités poétiques » (Orpustan, J.B., 1996: 190), mais vite popularisées, a aussi étudié le chant classique. C'est d'ailleurs avec un récital. d'airs d'opéras qu'il se produit à Bayonne en 1846: on est loin de l'image de l'artiste vagabond, guitare en bandoulière, tel que le représente P. Bringas dans un tableau devenu célèbre, et qui, avec ses zortziko ${ }^{32}$ à cinq temps, incarne un chant basque fort d'une particularité rythmique qu'il considère comme sa marque caractéristique. Ce sont sans doute ses chansons qui, les premières, portent dans leur ensemble texte/musique 
(que l'on ne peut, ici, séparer) une expression, voire une revendication de basquitude telle qu'on les trouvera par la suite développées dans la chanson engagée des années 1960. Là commence en effet une autre série d'avatars du «chant basque ", qui mènent à un rock basque à la fois divers et vivant, constituant l'une des composantes si variées de ce domaine en pleine expansion à l'orée du vingt-et-unième siècle.

\section{Bibliographie}

A.B., 1854, "Lamazou à Bayonne », in Le Courrier de Bayonne, $1^{\circ}$ octobre, $\mathrm{n}^{\circ} 249$.

ARANA MARTLA, J.A., 1983, «Iparraguirre y el folkJore vasco », in Folklore. Cuadernos de sección 1, SaintSébastien, Eusko Ikaskuntza : 121-128.

Aulnoy, Comtesse d', 1874 [1679], La cour et la ville de Madrid, Paris, Plon.

AVEZAC, d', 1841, " Basques ", in Encyclopédie norvelle ou dictionnaire philosophique, scientifique, littéraire et industriel, fac-simile Genève, Slatkine Reprints.

AzKuE, R.M. de, 1990 [1922], Cancionero popular vasco, Bilbao, Euskalzaindia, volume I. Baskonia, La, 1896, "Notas musicales », 20 octobre : 24

Benichou, P., 1970, Nerval et la chanson folklorique, Paris, Librairie José Corti.

BIDART, P., 1997, «L'aufklärung et la basquité » in Lapurdum II, UPRESA du CNRS/Université de Bordeaux IIU/Département d'Etudes Basques de Bayonne : 303-338

BLANCHARD, H., 1853, "Audition musicale », in Revue et gazette musicale, $1^{\text {T }}$ mai, $\mathrm{n}^{\circ} 18: 161$.

BORDES, C., 1899, « La musique populaire des Basques », in La Tradition au Pays Basque, fac-simile SaintSébastien, Elkar, 1982.

BorRow, G., 1989 [1842, traduction française 1845], La Bible en Espagne, Paris, Phébus.

Boucher de Crevecoeur, E., 1823, Souvenirs du Pays Basque ei des Pyrénées en 1819 et 1820, Paris, Goetschy.

CHAно, A., 1979 [1936], Voyage en Navarre pendant l'insurrection des Basques, fac-similé Marseille, Lafitte reprints.

CinAHo, A., 1844, "Chants basques ", in Ariel, 6 octobre, $n^{\circ} 1$.

Chaнo, A., 1845, «Poésie cantabre», in Ariel, 27 avril, $n^{\circ} 30 ; 4$ septembre, $n^{\circ} 50$.

CHAHO, A., [1855], Biarritz entre les Pyrénées et l'Océan. Itinéraire pittoresque, Bayonne, Lespès.

CHEYRONNAUD, J., (éd.) 1997, Instructions pour un recueil général de poésies populaires de la France (18521857), Paris, Editions du C.T.H.S.

Courrier de Bayonne, Le, 1853, «Fêtes d'Urrugne », 4 septembre, $n^{\circ} 137$.

Courrier de Bayonne, Le, 1856, [sans titre], 7 septembre, $n^{\circ} 537$.

Courrier de Bayonne, Le, 1860, [sans titre], 19 septembre, h ${ }^{\circ} 1160$.

Courrier de Bayonne, Le, 1862, "Espagne ", 5 septembre, $n^{\circ} 1463$.

Courrier de Bayonne, Le, 1866, [sans titre], 23 septembre, n'2092.

Courrier de Bayonne, Le, 1867, [sans titre], 25 septembre, $n^{\circ} 2245$.

Courrier de Bayonne, Le, 1867, [sans titre], 27 septembre, $n^{\prime} 2246$.

D., 1865, [sans titre], in Le Courrier de Bayonne, 22 septembre, $\mathrm{n}^{2} 1936$.

Dictionnaire universel Lamusse, 1867, article « Basques », Paris, Larousse.

Donostia, J.A., 1985 [1923], « Comment chante le Basque », in Obras completas, Saint-Sébastien, Eusko Ikaskuntza, volume IV.

DonostiA, J.A., 1931, « Essai d'une bibliographie musicale basque », in Bulletin du Musée Basque : 1-29.

DonostiA, J.A., 1923, "Quelques caractéristiques de la chanson basque », in Gure Herria : 677-689.

ECHAVE, A. de, 1910, «El teatro lírico vasco », in La Baskonia, 10 octobre, $\mathrm{n}^{\circ} 613$ : 3-9.

ETCHEBERR, 1636, Eliçara erabilceco liburua, Bordeaux., G. Millanges. 
Euskalerriaren Alde, 1911, « El Orfeón Donostiarra y la pastoral Mendi-Mendiyan » : 224-248.

FAURIEL, C., 1836, Histoire de la Gaule méridionale sous la domination des conquérants germains, Paris, Paulin.

FetIS, F.J., 2/1836, La musique mise à la portée de tout le monde, Paris, Paulin.

FETIS, F.J., 2/1878, Biographie universelle des musiciens, Paris, Firmin Didot.

FlaUBERT, G., 1964 [1857], Madame Bovary in CEures complètes, Paris, Seuil, tome I.

FORTOUL, H., 1841, « Chanson », in Encyclopédie nouvelle ou dictionnaire philosophique, scientifique, littéraire et industriel, fac-similé Genève, Slatkine Reprints, 1991.

FRANCISQUE-MICHEL, 1857, Le Pays Basque, sa population, sa langue, ses mours, sa littérature et sa musique, fac-similé Saint-Sébastien, Elkar, 1983.

Furet, Le, 1841, [Programme du concert], 26 septembre, ${ }^{\circ} 43$.

Garay de MonglaVE, E., 1/1833, 2/1873, « Basques », in Dictionnaire de la conversation et de la lecture, Paris, 1/Belin-Mandar, 2/Firmin Didot.

Guillaume-Reicher, G., 1933, "Un voyageur allemand en Pays Basque en. 1850 ", in Revue Internationale des Études Basques: 432-445.

Haritschelfar, J, 1969, Le poète souletin Pierre Topet-Etchahun, Bayonne, Société des Amis du Musée Basque.

HenRY, A., 1857, «Lettres à un ami », in Le Courrier de Bayonne, 27 septembre, nº 699.

HEREAU, E., 1/1833, 2/1878, "Chanson », in Dictionnaire de la conversation et de la lecture, Paris, 1/BelinMandar, 2/Firmin Didot.

Hugo, V, 1984 [1843], Les Pyrénées, Paris, La Découverte.

HumBolDT, W.F. von, traduction espagnole 1975 [1801], Los Vascos, Saint-Sébastien, Auñamendi,

IHARCE DE BIDASSOUET, abbé d', 1825, Histoire des Cantabres ou des premiers colons de toute l'Europe, avec celle des Basques, Paris, Jules Didot.

IzIUETA, J.I. de, 1973 [1826], Gipuzkoako dantzak, Saint-Sébastien, Eusko Ikaskuntza.

JEANPIERRE, H., 1965, «Bayonne, Biarritz et le Pays Basque dans la correspondance de Mérimée », in Bulletin de la Société des Sciences, Lettres et Arts de Bayonne, $\mathbf{n}^{\circ} 109: 381-436$.

JouY, E. de, 1818, L'Hermite en province ou observations sur les maurs et les usages français au commencement du xzx siècle, Paris, Pillet.

JuARISTI, J., 1987, El linaje de Aitor. La invención de la tradición vasca, Madrid, Taurus.

LABORDE, J.-B. de, 1780, Essai sur la musique ancienne et moderne, Paris, imp. de Ph.D.Pierres, tome III.

Lamazou, P., 3/1877, Cinquante chants pyrénéens, Paris, P. Lamazou.

LINK, F., 1801, Travels in Portugal and through France and Spain, Londres, T.N. Longman and O. Rees.

M., J. de, 1842, " Basques (Provinces) (géogr.) », in Encyclopédie catholique, répertoire universel et raisonné des sciences, des letires, des arts et des métiers, Paris, Parent-Desbarres.

Mabru, L., [s.d.], Pratiques collectives du chant traditionnel dans le sud de, la France, [s.1], Aram LR/Ministère de la Culture.

MASEIN, 1792, Essai historique sur la ville de Bayonne et de son commerce, Paris, chez. I'auteur / Bordeaux / Bayonne.

Mazure, A., 1839, Histoire du Béarn et du Pays Basque, Pau, E. Vignancour.

Mrer, 1841, "Sur Pierre-Jean Garat ", in La Sentinelle des Pyrénées [La France Musicale], 18 février, $n^{\circ} 1490$,

MonCLA, J., 1854, « Théâtre de Bayonne ", in Le Courrier de Bayonne, 5 octobre, $\mathrm{n}^{\circ} 250$.

MOREL, M.F., 1836, Bayonne, vues historiques et descriptives, Bayonne, Lamaignère.

OLIVIER, G., 1/1833, 2/1878, " Chants populaires », in Dictionnaire de la conversation et de la lecture, Paris, 1/Belin-Mandar, 2/Firmin Didot.

ORPuStan, J.-B., 1996, Précis d'histoire littéraire basque, Saint-Etienne-de-Baïgorry, Izpegi.

PARISOT et anonyme, 1855, "Fauriel », in Biographie universelle ancienne et moderne (Michaud), Paris, $\mathrm{M}^{\infty} \mathrm{C}$. Desplaces.

Pedrell, F., 1893, Pour notre musique, Barcelone, Juan Pujol \& Cia. 
RIEZU, J. de, (éd) 1994, « Papeles de Humboldt », in Obras completas del Padre Donostia, Saint-Sébastien, Eusko ikaskuntza, volume VIII.

Rousseau, J.-J., 1977 [1767] Dictionnaire de musique, Paris, Art et culture, tome I.

SallaberRY, J.D.J, 1870, Chants populaires du Pays Basque, Bayonne.

Sentinelle des Pyrénées, La, 1841, "Concert de M. Alard », 21 septembre, $\mathrm{n}^{\circ} 1588$.

SPACH, L., 1835, "Chants populaires», in Encyclopédie des gens du monde, répertoire universel des sciences, des lettres et des arts, Paris, Lib. de Treuttel et Würtz.

Stern, L., 1837, «Concert de la Société philharmonique », in Le Phare de Bayonne, 2 novembre, $\mathrm{n}^{\circ} 465$.

ThEESSE, A.M., 1999, La création des identités nationales, Paris, Seuil.

TIERSOT, J., 1909, «Charles Bordes et la chanson populaire », in Tribune de Saint-Gervais, numéro spécial : 20-26.

Tribly, 1844, [sans titre], 11 août, n 5 .

URKIZU, R., (éd.) 1987, Bertso zahar eta berri zerbaiten bilduma (1798), Durango, Durangoko udala.

Vulehel IO, M“ de la, 1869, Souvenirs des Pyrénées, [Paris, L. Parent].

Vinson, J., 1883, Le folklore du Pays Basque, Paris, Maisonneuve et C".

Vinson, J., 1.884, Notice bibliographique sur le folklore basque, Paris, Maisonneuve frères \& Leclerc.

VOLTAIRE, F.M., 1966 [1768], La princesse de Babylone, in Romans et contes, Paris, Garnier/Flammarion.

WALCKENAER, Baron de, 1834, «Basques (peuple et langage) », in Encyclopédie des gens du monde, réper-

toire universel et raisonné des sciences, des lettres et des arts, Paris, Lib. de Treuttel et Würtz.

WILKINSON, H., 1838, Sketches of scenery in the basque provinces of Spain, Londres, Ackermann \& Co.

Y., 1837, "Concert de la Société Philarmonique», in La Sentinelle des Pyrénées, 2 novembre, $\mathrm{n}^{\circ} 974$.

\section{Notes}

1. Un exemple, donné dans une histoire de Bayonne par P. Masein (1792: 86-87) : « les habitants des Pyrénées (...) sont en général d'une gaîté bondissante (...), leurs chansons, leurs entretiens expriment la vivacité de leur caractère, quoiqu'étant passionnés de fêtes, de la musique et de la danse, ils passent rapidement du tumulte dans le calme $m$.

2. On y verrait plutôt... le Basque, tel que le décrit le Père Donostia dans Comment chante le Basque: «le Basque chante, et il chante toujours et partout : à la maison, à l'église, dans la rue, à la campagne. Joyeux ou triste, il chante quand même " (1985 [1923] : 117).

3. Cité par P. Urkizu en exergue de Bertso zahar eta berri zenbaiten bilduma (1798).

4. De même, lorsque le botaniste Moritz Wilkomm visite le Pays Basque en 1850 , il prête une attention particulière aux pratiques vocales qu'il rencontre : enfants chantant en chœur, sur une « mélodie spéciale et monotone ", zortziko dont il donne les paroles basques et la traduction allemande, et qu'il accompagne de commentaires (Guillaumie-Reicher, G., $1933: 432-442$ ).

5. Zortziko : «à huit », sans doute en référence au nombre de vers ou de pas puisqu'il s'agit aussi d'une forme poétique et d'une danse, mais correspondant à une mesure à 5 temps, longtemps notée à $6 / 8$, puis à $5 / 8$, avec une succession régulière de fragments à $3 / 8$ et à $2 / 8$.

6. J. Michelet n'a malheureusement pas mené à bien le projet d’une Encyclopédie des chants populaires, conçue vers 1828-1830 à l'exemple du recueil d'Herder, et qui aurait sans doute compris des « chants populaires " des Basques, puisqu'il mentionne ceux-ci dans son cours à l'Ecole Normale (Bénichou, P., 1970: 151).

7. Voir l'étude qu'en font Paul Bénichou (1970: 47 et ss) et Anne-Marie Thiesse (1999: :50-59).

8. On trouvera les textes relatifs à l'organisation, à la méthode utilisée et préconisée, ainsi qu'au dépouillement des réponses de cette vaste enquête diligentée par le ministre de l'instruction publique H. Fortoul dans l'ouvrage édité et introduit par J. Cheyronnaud, Instructions pour un Recueil général de poésies populaires de la France (1852-1857). C'est J.J. Ampère qui rédige les Instructions proprement dites.

9. En version moderne et castillane, prenant la place d'une diffusion historique véritable, et avec une audience élargie à toute $1^{\prime} E s p a g n e$, comme le montre Jon Juaristi (1987). 
10. Cité par J. Haritschelhar (1969: 386).

11. Julie Adrienne Carricaburu, auteur de l'un des premiers recueils imprimés de chants basques (voir plus loin).

12. On peut en suivre les principale étapes dans la Notice bibliographique sur le folklore basque de J. Vinson (1884: 29-56) : Garay de Monglave, Chaho, Antoine d'Abbadie, du Mège, Fauriel, Francisque Michel, Mary-Lafon, Bladé, Cénac-Moncaut en sont les principaux protagonistes.

13. Cité par Francisque-Michel ( 1857 : 213). Cette idée ne figure pas exactement sous cette forme dans l'édition française utilisée pour les autres citations de G. Borrow, qui est basée sur la troisième édition anglaise de l'ouvrage, légèrement différente de la première.

14. « Peuple bizarre, qui, jeté, comme un monument antique, entre la France et l'Espagne, entre les Pyrénées et l'Océan, semble, par ses mœurs, sa langue et ses usages, séquestré du monde entier, étranger au bouleversement des empires et stationnaire à cóté des progrès de la civilisation » dit $\mathrm{E}$. Garay de Monglave dans le Dictionnaire de la conversation (1833 : article « Basques »).

15. Et il est le premier à consacrer, en langue française, un (court) chapitre à la musique basque, instrumentale et vocale.

16. Inconnu des dictionnaires musicaux. S'agit-il d'un parent de Léonard Amé, que F.J. Fétis dit avoir été flûtiste au Théâtre de la Gaîtè entre 1814 et 1823 ? (Fétis, F.J., 1878 : article " Amé »).

17. Quelques années plus tard, une jeune Anglaise, Louisa Stuart Costello, cite et traduit plusieurs chansons basques dans le récit du voyage qu'elle a effectué en 1842, mais sans en donner la musique (Bearn and the Pyrenees, Londres, 1844).

18. Air basque varié avec introduction pour le violon, avec accompagnement de forte-piano ou deux violons, alto et basse.

19. Comme l'avait fait, bien plus tôt, la danse basque, qui a inspiré plusieurs œuvres instrumentales : Branle de Basque de Louis Couperin (Second livre d'orgue, 1656), Le Basque de Marin Marais (Cinq vieilles danses françaises, s. d), Le Basque de François Couperin (Second livre de pièces de clavecin, 1717), Le Basque de Josse Bioutmy (Premier livre de pièces de clovecin, 1738), Le Basque de Jean-Baptiste Dupuits Pièces de caractère pour la vielle, s. d), jusqu'aux Basquaises d'Adolphe Adam (La filleule des fées, 1849).

20. Il s'agit de Mendian zoinen eder dont il a été question à propos de Garat,

21. Les cantiques basques connaítront une publication plus précoce, tant pour les textes (depuis Jean Etcheberri de Ciboure: Noelac eta berce canta espiritual berriac, 1631) que pour la musique (Cantica izpiritualak, 1857)

22. Paul Lacôme d'Estalenx, compositeur et critique musical, né en 1838 au Houga (Gers). Il sera également l'éditeur d'Echos d'Espagne (chansons et darises populaires recueillies et transcrites par P. Lacome et J. Puig Absubide), d'un recueil d'arrangements pour piano intitulé Album pyrénéen el du Tour du Monde en 10 chansons nationales et caractéristiques.

23. Cent numéros paraissent à Saint-Sébastien avant 1878, édités par A. Diaz. Signalons aussi que beaucoup de ces airs basques sont de composition récente (Donostia, J.A., 1931 : 19).

24. Par exemple, Donostiako iru damatxo, dont il est question à plusieurs reprises, devient dans le recueil de P. Lamazou Les Donzelles de Saint-Sébastien.

25. Autre Georges Amé, dont nous n'avons pas retrouvé de trace...

26. Notons aussi que "deux ans plus tôt, Georges Bizet publiait un recueil de « six mélodies populaires avec accompagnement de piano » intitulé Chant des Pyrénées, contenant des mélodies béarnaises.

27. Signalons aussi la " mazurka de salon » pour piano de I.A. Santesteban, compositeur né à SaintSébastien, publiée à Paris en 1856 et intitulée Souvenir de mon pays, mais qui ne semble pas être basée sur des airs basques.

28. Publications que l'on peut compléter par le recueil inédit réuni avant 1839 par Gaston Sacaze.

29. Seules 5 chansons seront effectivement éditées, mais Bordes mènera à bien d'autres publications de chants basques.

30. Démarche qui prend tout son sens si I'on songe à l'action de Bordes en faveur du renouveau du chant grégorien et de la musique palestrinienne.

31. On a prononcé le nom d'Hayet au sujet du ténor dont se serait inspiré Flaubert : originaire de Biarritz, formé au Canservatoire de Paris auprès de Révial, il débutera à l'Opéra de Paris en 1861 .

32. Spécifícité basque qui n'en est pas une, puisque Braïloïu ou Bartok ont montré: l'existence de mesures similaires en Turquie ou en Europe centrale : néanmoins le zortziko est considéré, surtout dans les provinces basques méridionales - et en partie devenu-comme la signature de la musique basque, tant, vocale qu'instrumentale. 